\title{
Room Temperature, Open-Flask C-P Bond-Formation on Water under Catalyst-Free Conditions
}

\author{
Rakhee Choudhary $\ddagger$ \\ Rekha Bai ${ }^{\ddagger}$ \\ Pratibha Singh ${ }^{\ddagger}$ \\ Mahesh C. Sharma \\ Satpal Singh Badsara* (1)
}

MFOS Laboratory, Department of Chemistry (Centre of Advanced Study), University of Rajasthan, JLN Marg, Jaipur, Rajasthan-302004, India

badsarass4@uniraj.ac.in

sattubhu2005@gmail.com

${ }^{\ddagger}$ Authors contributed equally
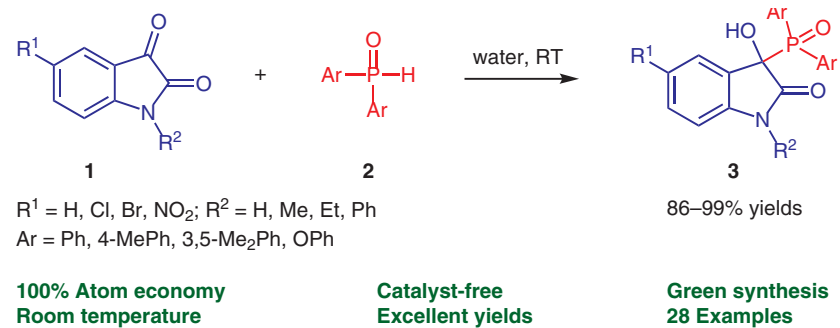

Green synthesis 28 Examples

\author{
Received: 31.03.2018 \\ Accepted after revision: 08.05.2018 \\ Published online: 13.06 .2018 \\ DOI: 10.1055/s-0037-1610167; Art ID: so-2018-d0026-op
}

License terms: (c)

Abstract A catalyst-free C-P bond-formation in an open flask at room temperature between isatin derivatives and phosphorus surrogates on water is described. Isatin derivatives possessing different substitutes underwent C-P coupling reaction with a variety of phosphine oxides under the reaction conditions employed, providing the desired products in up to quantitative yields.

Key words catalyst-free, $\mathrm{C}-\mathrm{P}$ bond formation, isatin

Organophosphorus compounds play important roles in organic synthesis, ${ }^{1}$ organometallic chemistry, ${ }^{2}$ medicinal chemistry, ${ }^{3}$ chemical biology, ${ }^{4}$ and material science. ${ }^{5}$ More specifically, $\alpha$-hydroxy- and $\alpha$-aminophosphonic acids have roles as biophosphate mimics, antibiotics, antivirals, and antitumor agents. ${ }^{6}$ Nucleophilic substitution of toxic phosphinoyl halides with organometallic reagents has been the traditional method for the synthesis of these compounds. ${ }^{1 \mathrm{~d}}$ Subsequently, the Hirao transition-metal-catalysed phosphonation of organohalides has emerged as a facile alternative. ${ }^{7}$ Use of toxic phosphorus halides, high catalyst loading, harsh reaction conditions or poor functional group tolerance are drawbacks of these approaches.

Oxindole frameworks bearing a C-3 quaternary stereocenter are important constituents of many natural products and biologically active molecules. ${ }^{8}$ These molecules are often synthesized via aldol reactions of aldehydes or ketones or other nucleophilic species to the 3-carbonyl of isatins. ${ }^{9}$

In recent years, organophosphorus compounds have been mostly synthesized via cross-coupling reactions, ${ }^{10} \mathrm{C}-\mathrm{H}$ activation, ${ }^{11}$ and dehydrogenative coupling reactions. ${ }^{12} \mathrm{Sev}-$ eral catalytic systems such as the $\mathrm{NHC} / \mathrm{Icy} . \mathrm{CO}_{2}$ precatalyst, organocatalysts, and palladium pincer complexes have been employed for phospha-Michael additions to activated alkenes and alkynes. ${ }^{13}$ Recently, Cai and co-workers disclosed a copper catalyst with fluorous bis(oxazoline) as a ligand for the asymmetric $\alpha$-hydrophosphonylation of isatins. ${ }^{14 a}$ Swamy and co-workers have reported a catalyst-free addition of allenyl or alkynyl-phosphonates and phosphine oxides. ${ }^{14 \mathrm{~b}}$ Although various synthetic pathways using different catalytic systems have been developed for the preparation of $\alpha 1$-oxindole- $\alpha$-hydroxy phosphonates, ${ }^{15,16}$ the synthesis of oxindoles containing $\alpha$-hydroxy phosphinoyl compounds under mild conditions remains a major goal. As a part of our ongoing project to develop metal-free organic transformations, ${ }^{17}$ we have developed a facile and efficient synthetic protocol for preparation of oxindoles containing an $\alpha$-hydroxyphosphinoyl group by a catalyst-free C-P bond formation between isatins and phosphine oxides or phosphites.

For optimization studies, we choose isatin 1a and diphenylphosphine oxide $\mathbf{2 a}$ as model substrates. Initially, the $\mathrm{C}-\mathrm{P}$ coupling was carried out in toluene at $100{ }^{\circ} \mathrm{C}$ to afford the desired $\alpha$-hydroxyphosphinoyl oxindole 3a in 95\% yield after $12 \mathrm{~h}$ (Table 1 , entry 1 ). When the reaction was carried out at room temperature in toluene no improvement in yield was observed (entry 2). A slight improvement in yield (96\%) was observed when the reaction was carried out in isopropanol as the solvent (entry 3), whereas C-P coupling reaction in the absence of solvent at ambient or elevated temperature led to lower yields (entries 4 and 5). Remarkably, the reaction between $\mathbf{1 a}$ and $\mathbf{2 a}$ proceeded smoothly and efficiently in water at room temperature, affording $\mathbf{3 a}$ in excellent yield (98\%). 
Table 1 Optimization of Reaction Conditions for C-P Bond Formation between Isatin $\mathbf{1 a}$ and Diphenylphosphine Oxide $\mathbf{2} \mathrm{a}^{\mathrm{a}}$

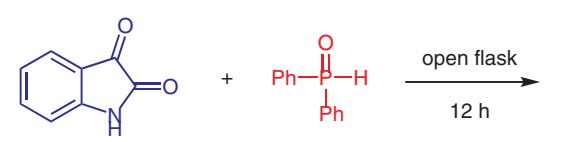

1a

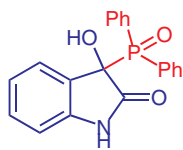

$3 a$

\begin{tabular}{lllll}
\hline Entry & Solvent & Temperature $\left({ }^{\circ} \mathrm{C}\right)$ & Time $(\mathrm{h})$ & Yield $(\%)^{\mathrm{b}}$ \\
\hline 1 & toluene & 100 & 12 & 95 \\
2 & toluene & RT & 24 & 90 \\
3 & $i$-PrOH & RT & 12 & 96 \\
4 & neat & 100 & 24 & 74 \\
5 & neat & RT & 24 & 58 \\
6 & water & RT & 12 & 98
\end{tabular}

a Reaction conditions: Isatin $\mathbf{1 a}(0.5 \mathrm{mmol})$, diphenylphosphine oxide $\mathbf{2 a}$ $(0.5 \mathrm{mmol})$, solvent or neat, r.t. to $100{ }^{\circ} \mathrm{C}$.

${ }^{\mathrm{b}}$ Isolated yields based on $\mathbf{1 a}$.

After optimization studies, we then turned our attention to study the substrate scope of this intriguing C-P bond formation. For this, isatin 1a and $\mathrm{N}$-alkylated isatins $\mathbf{1 b}-\mathbf{k}$ (prepared by following a known procedure ${ }^{18}$ ) were employed for the C-P cross-coupling reaction with diphenylphosphine oxide $\mathbf{2 a}$ on water under the optimized reaction conditions. The corresponding oxindole containing $\alpha$-hydroxyphosphinoyl compounds $\mathbf{3 b} \mathbf{b}-\mathbf{k}$ were obtained in 9199\% yields (Scheme 1). Other phosphine oxides $\mathbf{2 b}$ and $\mathbf{2 c}$ also reacted well with different isatins to provide the desired products $\mathbf{3 1 - \mathbf { x }}$ in $\mathbf{8 6 - 9 6 \%}$ yields. The structures of all the compounds $\mathbf{3 a}-\mathbf{x}$ were established based on ${ }^{1} \mathrm{H}$ NMR, ${ }^{13} \mathrm{C}$ NMR, ${ }^{31} \mathrm{P}$ NMR spectroscopy and HRMS analysis.

We next attempted the synthesis of $\alpha 1$-oxindole- $\alpha$-hydroxyphosphonates using this interesting C-P bond-formation methodology. Accordingly, we employed diphenyl phosphite $\mathbf{2 d}$ for $\mathrm{C}-\mathrm{P}$ bond formation with isatin derivatives $\mathbf{1 a}-\mathbf{c}$ and $\mathbf{1 g}$ under neat conditions at room temperature. Under these conditions, efficient coupling provided the desired $\alpha 1$-oxindole- $\alpha$-hydroxyphosphonates 3y1-3y4 in 86$93 \%$ yields (Scheme 1 ).

To elucidate the reaction mechanism, we performed several control experiments as shown in Scheme 2. Initially, assuming that the reaction with diphenylphosphine oxide proceeded following a radical pathway, ${ }^{13}$ the reaction between $\mathbf{1 b}$ and $\mathbf{2 a}$ was carried out in the presence of the free radical quencher 2,2,6,6-tetramethylpiperidine-1-oxyl (TEMPO; Scheme 2, equiv 1), but we found that there was no effect on the reaction as the desired product 3a was obtained in $97 \%$ yield. We also performed the reaction between TEMPO and $\mathbf{2 a}$ (Scheme 2, equiv 2), TEMPO and $\mathbf{1 b}$ (Scheme 1, equiv 3 ) and TEMPO and $\mathbf{2 d}$ (Scheme 2, equiv 4 ). However, no coupled product was observed in these cases. These results clearly rule out the possibility of a radical pathway during the formation of products 3 .

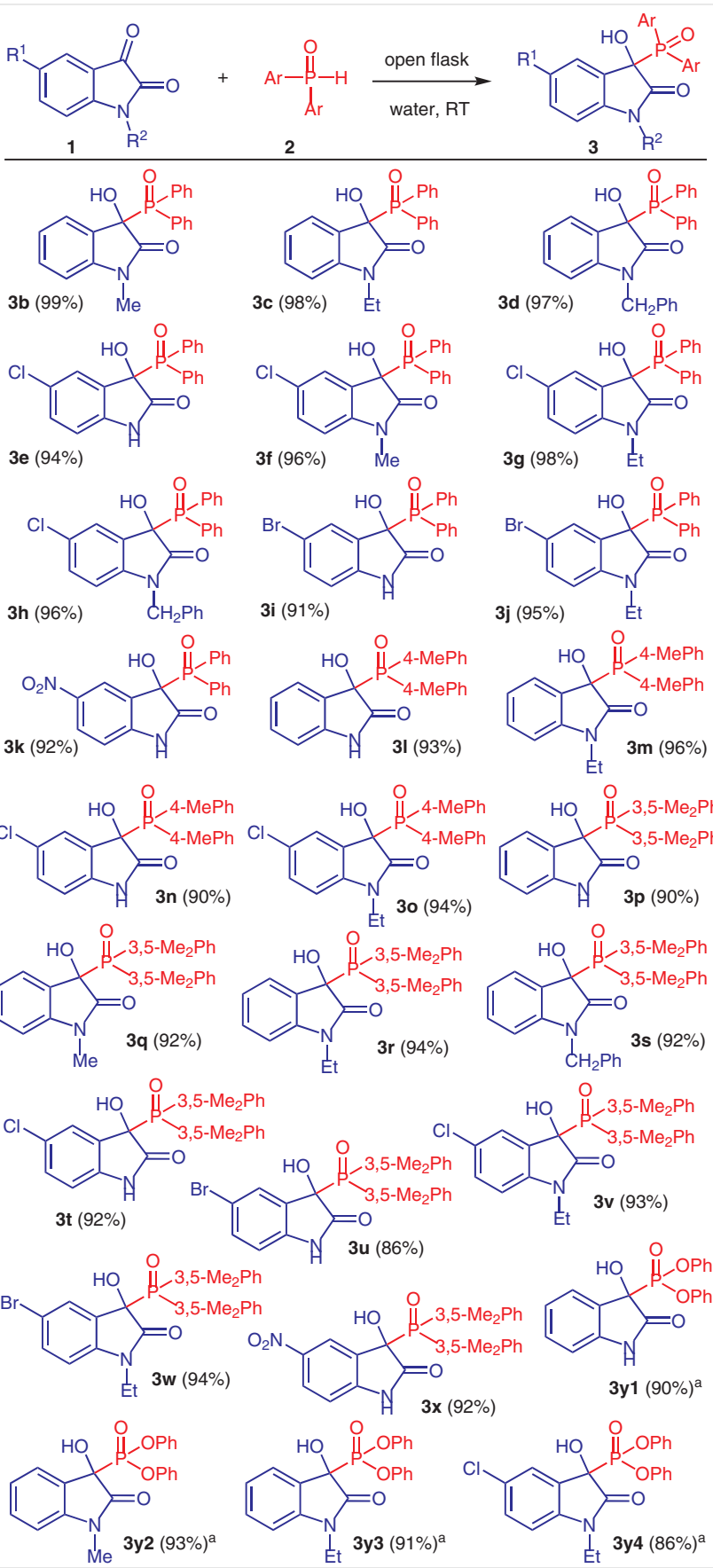

Scheme 1 C-P bond formation between isatins 1 and phosphine oxides 2 . Reaction conditions: Isatin $\mathbf{1}(0.5 \mathrm{mmol})$, diphenylphosphine oxide $2(0.5 \mathrm{mmol})$, water $(1.0 \mathrm{~mL})$, r.t., $12 \mathrm{~h}$. Isolated yields are based on 1. ${ }^{\text {a }}$ Reaction conditions: Isatin $\mathbf{1}(1.0 \mathrm{mmol})$, diphenyl phosphite $\mathbf{2 d}$ $(0.2 \mathrm{~mL})$, neat, r.t., $12 \mathrm{~h}$.

A literature survey revealed that diarylphosphine oxides 2 in the presence of air can undergo tautomerism to generate the corresponding phosphinous acid $\mathbf{2}^{\prime} \cdot{ }^{19}$ On this basis, a plausible reaction mechanism for this interesting catalyst- 

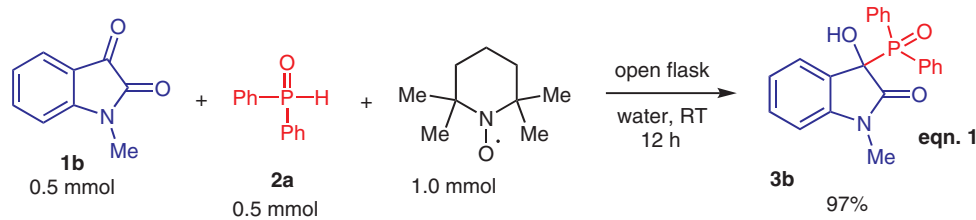

$$
\begin{gathered}
\text { O } \\
\text { Ph-P } \\
\text { II } \\
\text { Ph }
\end{gathered}
$$<smiles>CC1(C)CCCC(C)(N)N1[O-]</smiles>

$$
\underset{\text { water, RT }}{\stackrel{\text { open flask }}{\longrightarrow}}
$$

No reaction

$2 \mathrm{a}$
$0.5 \mathrm{mmol}$

$1.0 \mathrm{mmol}$<smiles>CN1C(=O)C(=O)c2ccccc21</smiles><smiles>CC1(C)CCCC(C)(C)N1[O]</smiles>

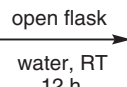

No reaction

eqn. 3

$12 \mathrm{~h}$<smiles>O=P(O)(O)c1ccccc1</smiles><smiles>CC1(C)CCCC(C)(C)N1[O-]</smiles>

$\underset{\substack{\text { water, RT } \\ 12 \mathrm{~h}}}{\stackrel{\text { open flask }}{\longrightarrow}}$

No reaction 2d

$0.5 \mathrm{mmol}$

$1.0 \mathrm{mmol}$

Scheme 2 Control experiments

free C-P bond formation is depicted in Scheme 3 for the reaction between $\mathbf{1}$ and $\mathbf{2}$. Initially phosphine oxide $\mathbf{2}$ converts into its tautomer $\mathbf{2}^{\prime}$. Then $\mathbf{2}$ ' attacks the electrophilic $\mathrm{C}=\mathrm{O}$ of isatin $\mathbf{1}$ to generate intermediate $\mathbf{A}$, which is subsequently converted into the product $\mathbf{3}$.

In conclusion, we have reported a convenient protocol for the synthesis of oxindole $\alpha$-hydroxyphosphinoyl compounds and $\alpha 1$-oxindole- $\alpha$-hydroxyphosphonates via a catalyst-free C-P bond formation between isatins and phosphine oxides or phosphites on aqueous medium. The corresponding products were obtained in excellent yields.

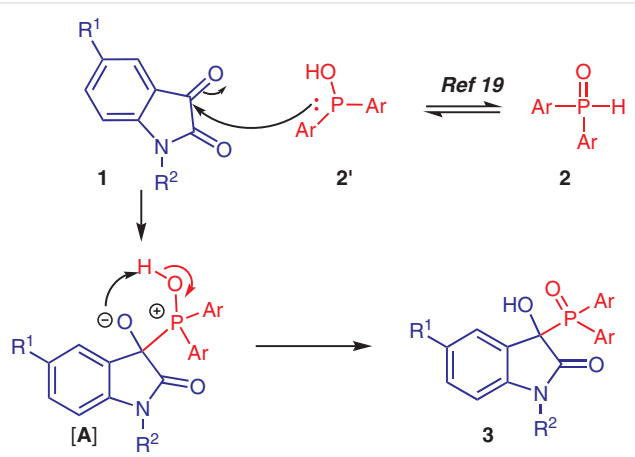

Scheme 3 Plausible reaction mechanism for C-P bond formation between isatin and diphenyl phosphine oxide

All chemicals were purchased from commercial suppliers and used without further purification. NMR spectra were recorded with a JEOL Ressonance-400 instrument using $\mathrm{CDCl}_{3}$ or DMSO- $d_{6}$ as solvent. In some cases, one drop of DMSO- $d_{6}$ was added to $\mathrm{CDCl}_{3}$ to improve solubility. Chemical shifts are reported in parts per million (ppm) and referenced to the residual solvent resonance. Coupling constants $(J)$ are reported in Hertz $(\mathrm{Hz})$. Standard abbreviations indicating multiplicity are used as follows: $\mathrm{s}=$ singlet, $\mathrm{d}=$ doublet, $\mathrm{t}=$ triplet, $\mathrm{dd}=$ double doublet, $\mathrm{dt}=$ double triplet, $\mathrm{q}=$ quartet, $\mathrm{m}=$ multiplet. HRMS data were collected with a Waters - Xevo G2S QTof LC-MS with UPLC.

\section{General Procedure for Table 1}

3-(Diphenyl-phosphinoyl)-3-hydroxy-indolin-2-one (3a)

Diphenylphosphine oxide $\mathbf{2 a}(0.5 \mathrm{mmol}, 101 \mathrm{~g})$ was added to stirred isatin $1 \mathrm{a}(0.5 \mathrm{mmol}, 0.073 \mathrm{~g})$ in an open flask in solvent $(1.0 \mathrm{~mL})$ or neat and then the reaction mixture was stirred at the given temperature. The mixture was then diluted with EtOAc $(10 \mathrm{~mL})$. After usual workup, the organic layer was dried over $\mathrm{Na}_{2} \mathrm{SO}_{4}$, filtered, EtOAc was evaporated, and the crude product thus obtained was purified by crystallization (EtOAc) to afford the corresponding product $\mathbf{3 a}$ as a white solid. Yield: $0.171 \mathrm{~g}(98 \%) ; \mathrm{mp} 142{ }^{\circ} \mathrm{C}$.

${ }^{1} \mathrm{H}$ NMR (400 MHz, DMSO- $d_{6}$ ): $\delta=6.38(\mathrm{~d}, J=7.2 \mathrm{~Hz}, 1 \mathrm{H}), 6.72(\mathrm{~s}$, $1 \mathrm{H}), 6.75$ (dd, $J=3.2,7.6 \mathrm{~Hz}, 1 \mathrm{H}), 7.18(\mathrm{t}, J=7.6 \mathrm{~Hz}, 1 \mathrm{H}), 7.25-7.32$ $(\mathrm{m}, 1 \mathrm{H}), 7.41-7.59(\mathrm{~m}, 5 \mathrm{H}), 7.63(\mathrm{t}, J=7.6 \mathrm{~Hz}, 1 \mathrm{H}), 7.85-8.02(\mathrm{~m}$, $4 \mathrm{H}), 10.49$ (s, $1 \mathrm{H})$.

${ }^{13} \mathrm{C}$ NMR $\left(100 \mathrm{MHz}\right.$, DMSO- $\left.d_{6}\right): \delta=79.8\left(\mathrm{~d}, J_{\mathrm{C}-\mathrm{P}}=82.1 \mathrm{~Hz}\right), 109.5$, $121.0,125.5,126.8,127.8\left(\mathrm{~d}, J_{\mathrm{C}-\mathrm{P}}=11.5 \mathrm{~Hz}\right), 128.1\left(\mathrm{~d}, J_{\mathrm{C}-\mathrm{P}}=11.3 \mathrm{~Hz}\right)$, $129.8,130.5\left(\mathrm{~d}, J_{\mathrm{C}-\mathrm{P}}=95.0 \mathrm{~Hz}\right), 130.7\left(\mathrm{~d}, J_{\mathrm{C}-\mathrm{P}}=95.2 \mathrm{~Hz}\right), 131.6,132.1$, $132.3\left(\mathrm{~d}, J_{\mathrm{C}-\mathrm{P}}=8.5 \mathrm{~Hz}\right), 142.9\left(\mathrm{~d}, J_{\mathrm{C}-\mathrm{P}}=5.9 \mathrm{~Hz}\right), 175.3$.

${ }^{31} \mathrm{P}$ NMR $\left(162 \mathrm{MHz}\right.$, DMSO- $\left.d_{6}\right): \delta=28.8$.

HRMS: $m / z[\mathrm{M}+\mathrm{H}]$ calcd. for $\mathrm{C}_{20} \mathrm{H}_{16} \mathrm{NO}_{3} \mathrm{P}+\mathrm{H}$ : 350.0946; found: 350.0941 . 


\section{General Procedure for Scheme 1}

Phosphine oxide $\mathbf{2}(0.5 \mathrm{mmol})$ was added to a stirred mixture of isatin $\mathbf{1 b}-\mathbf{x}(0.5 \mathrm{mmol})$ in water $(1.0 \mathrm{~mL})$ in an open flask at r.t. The stirring was continued for $12 \mathrm{~h}$, then the reaction mixture was diluted with EtOAc $(10 \mathrm{~mL})$. After usual workup, the organic layer was dried over $\mathrm{Na}_{2} \mathrm{SO}_{4}$, filtered, the EtOAc was evaporated and the crude product thus obtained was purified by crystallization (EtOAc or EtOAc/methanol) to afford the corresponding products $\mathbf{3 b}-\mathbf{x}$.

\section{For Compounds 3y1-y4}

Isatin $\mathbf{1}(1.0 \mathrm{mmol})$ and diphenyl phosphite $\mathbf{2 d}(0.2 \mathrm{~mL})$ were reacted neat in an open flask at r.t. for $12 \mathrm{~h}$ and the reaction mixture was then diluted with EtOAc (10 mL). After usual workup, the organic layer was dried over $\mathrm{Na}_{2} \mathrm{SO}_{4}$, filtered, the EtOAc was evaporated and the crude product thus obtained was purified by column chromatography (EtOAc/hexanes, 1:1) to afford the corresponding products 3y1-y4.

\section{3-(Diphenyl-phosphinoyl)-3-hydroxy-1-methylindolin-2-one (3b)}

The title compound was prepared by following the general procedure for Scheme 1, using $N$-methylisatin $\mathbf{1 b}(0.5 \mathrm{mmol}, 0.080 \mathrm{~g})$ and diphenylphosphine oxide $\mathbf{2 a}(0.5 \mathrm{mmol}, 0.101 \mathrm{~g})$, providing $\mathbf{3 b}$ as a white solid. Yield: $0.179 \mathrm{~g}(99 \%) ; \mathrm{mp} 130^{\circ} \mathrm{C}$.

${ }^{1} \mathrm{H}$ NMR (400 MHz, DMSO- $\left.d_{6}\right): \delta=2.99(\mathrm{~s}, 3 \mathrm{H}), 6.52(\mathrm{~d}, J=7.6 \mathrm{~Hz}$, $1 \mathrm{H}), 6.85(\mathrm{t}, J=7.6 \mathrm{~Hz}, 1 \mathrm{H}), 6.92(\mathrm{~d}, J=7.6 \mathrm{~Hz}, 1 \mathrm{H}), 7.29(\mathrm{t}, J=7.6 \mathrm{~Hz}$, $1 \mathrm{H}), 7.42-7.49(\mathrm{~m}, 2 \mathrm{H}), 7.51-7.59(\mathrm{~m}, 3 \mathrm{H}), 7.65(\mathrm{t}, J=7.6 \mathrm{~Hz}, 1 \mathrm{H})$, $7.83(\mathrm{~d}, J=7.6 \mathrm{~Hz}, 1 \mathrm{H}), 7.86(\mathrm{~d}, J=7.6 \mathrm{~Hz}, 1 \mathrm{H}), 7.97(\mathrm{~d}, J=7.6 \mathrm{~Hz}$, $1 \mathrm{H}), 7.99$ (d, $J=7.6 \mathrm{~Hz}, 1 \mathrm{H})$.

${ }^{13} \mathrm{C}$ NMR $\left(100 \mathrm{MHz}\right.$, DMSO- $\left.d_{6}\right): \delta=26.2,79.7\left(\mathrm{~d}, J_{\mathrm{C}-\mathrm{P}}=81.3 \mathrm{~Hz}\right), 108.4$, $121.8,125.2\left(\mathrm{~d}, J_{\mathrm{C}-\mathrm{P}}=2.7 \mathrm{~Hz}\right), 126.0,127.8\left(\mathrm{~d}, J_{\mathrm{C}-\mathrm{P}}=11.5 \mathrm{~Hz}\right), 128.3(\mathrm{~d}$, $\left.J_{\mathrm{C}-\mathrm{P}}=11.3 \mathrm{~Hz}\right), 129.8,130.0,130.7,131.8\left(\mathrm{~d}, J_{\mathrm{C}-\mathrm{P}}=1.6 \mathrm{~Hz}\right), 132.2\left(\mathrm{~d}, J_{\mathrm{C}-}\right.$ $\left.{ }_{\mathrm{P}}=8.8 \mathrm{~Hz}\right), 132.4\left(\mathrm{~d}, J_{\mathrm{C}-\mathrm{P}}=8.7 \mathrm{~Hz}\right), 144.0\left(\mathrm{~d}, J_{\mathrm{C}-\mathrm{P}}=5.6 \mathrm{~Hz}\right), 173.7$.

31P NMR (162 MHz, $\mathrm{CDCl}_{3} / \mathrm{DMSO}_{-} \mathrm{d}_{6}$ ): $\delta=30.3$.

HRMS: $m / z[\mathrm{M}+\mathrm{H}]$ calcd. for $\mathrm{C}_{21} \mathrm{H}_{18} \mathrm{NO}_{3} \mathrm{P}+\mathrm{H}$ : 364.1103; found: 364.1109 .

\section{3-(Diphenyl-phosphinoyl)-3-hydroxy-1-ethylindolin-2-one (3c)}

The title compound was prepared by following the general procedure for Scheme 1, using N-ethylisatin 1c $(0.5 \mathrm{mmol}, 0.087 \mathrm{~g})$ and diphenylphosphine oxide $\mathbf{2 a}(0.5 \mathrm{mmol}, 0.101 \mathrm{~g})$, providing $3 \mathbf{c}$ as a white solid. Yield: $0.184 \mathrm{~g}(98 \%) ; \mathrm{mp} 120^{\circ} \mathrm{C}$.

${ }^{1} \mathrm{H}$ NMR $\left(400 \mathrm{MHz}, \mathrm{CDCl}_{3}\right): \delta=0.85(\mathrm{t}, J=7.2 \mathrm{~Hz}, 3 \mathrm{H}), 3.28-3.33(\mathrm{~m}$, $1 \mathrm{H}), 3.52-3.57(\mathrm{~m}, 1 \mathrm{H}), 5.41(\mathrm{~d}, J=4.0 \mathrm{~Hz}, 1 \mathrm{H}), 6.62(\mathrm{~d}, J=7.6 \mathrm{~Hz}$, $1 \mathrm{H}), 6.87(\mathrm{t}, J=7.6 \mathrm{~Hz}, 1 \mathrm{H}), 7.10(\mathrm{~d}, J=7.6 \mathrm{~Hz}, 1 \mathrm{H}), 7.26(\mathrm{t}, J=7.6 \mathrm{~Hz}$, $1 \mathrm{H}), 7.31-7.39$ (m, $2 \mathrm{H}), 7.41-7.54(\mathrm{~m}, 3 \mathrm{H}), 7.55-7.71(\mathrm{~m}, 3 \mathrm{H}), 7.97$ (d, $J=7.6 \mathrm{~Hz}, 1 \mathrm{H}), 8.00(\mathrm{~d}, J=7.6 \mathrm{~Hz}, 1 \mathrm{H})$.

${ }^{13} \mathrm{C}$ NMR $\left(100 \mathrm{MHz}, \mathrm{CDCl}_{3}\right): \delta=12.0,34.9,80.2\left(\mathrm{~d}, J_{\mathrm{C}-\mathrm{P}}=71.3 \mathrm{~Hz}\right)$, $108.1,122.6,124.6,127.1,128.0\left(\mathrm{~d}, J_{\mathrm{C}-\mathrm{P}}=11.8 \mathrm{~Hz}\right), 128.2,128.4(\mathrm{~d}$, $\left.J_{\mathrm{C}-\mathrm{P}}=11.9 \mathrm{~Hz}\right), 129.1,130.3,132.4\left(\mathrm{~d}, J_{\mathrm{C}-\mathrm{P}}=9.1 \mathrm{~Hz}\right), 132.6,133.1(\mathrm{~d}$, $\left.J_{\mathrm{C}-\mathrm{P}}=8.8 \mathrm{~Hz}\right), 143.2\left(\mathrm{~d}, J_{\mathrm{C}-\mathrm{P}}=5.7 \mathrm{~Hz}\right), 173.5$.

${ }^{31} \mathrm{P}$ NMR $\left(162 \mathrm{MHz}, \mathrm{CDCl}_{3}\right): \delta=30.3$.

HRMS: $m / z[\mathrm{M}+\mathrm{H}]$ calcd. for $\mathrm{C}_{22} \mathrm{H}_{20} \mathrm{NO}_{3} \mathrm{P}+\mathrm{H}$ : 378.1259; found: 378.1245 .

\section{3-(Diphenyl-phosphinoyl)-3-hydroxy-1-benzylindolin-2-one (3d)} The title compound was prepared by following the general procedure for Scheme 1, using $N$-benzylisatin 1d $(0.5 \mathrm{mmol}, 0.118 \mathrm{~g})$ and diphenylphosphine oxide $2 \mathrm{a}(0.5 \mathrm{mmol}, 0.101 \mathrm{~g})$, providing $3 \mathbf{d}$ as a white solid. Yield: $0.213 \mathrm{~g}(97 \%) ; \mathrm{mp} 142^{\circ} \mathrm{C}$.
${ }^{1} \mathrm{H}$ NMR $\left(400 \mathrm{MHz}, \mathrm{CDCl}_{3}\right): \delta=4.53$ and $4.79(\mathrm{ABq}, J=15.6 \mathrm{~Hz}, 2 \mathrm{H})$, 4.66 (bs, $1 \mathrm{H}), 6.55$ (d, J = 7.6 Hz, $1 \mathrm{H}), 6.85$ (t, J = 7.6 Hz, $1 \mathrm{H}), 6.93$ (d, $J=7.6 \mathrm{~Hz}, 1 \mathrm{H}), 7.06-7.10(\mathrm{~m}, 2 \mathrm{H}), 7.17(\mathrm{t}, J=7.6 \mathrm{~Hz}, 1 \mathrm{H}), 7.20-7.26$ $(\mathrm{m}, 3 \mathrm{H}), 7.32-7.65(\mathrm{~m}, 6 \mathrm{H}), 7.76(\mathrm{~d}, J=8.4 \mathrm{~Hz}, 1 \mathrm{H}), 7.79(\mathrm{~d}, J=$ $8.4 \mathrm{~Hz}, 1 \mathrm{H}), 7.97$ (d, J = 8.4 Hz, $1 \mathrm{H}), 7.99$ (d, $J=8.4 \mathrm{~Hz}, 1 \mathrm{H})$.

${ }^{13} \mathrm{C}$ NMR $\left(100 \mathrm{MHz}\right.$, DMSO- $\left.d_{6}\right): \delta=42.9,79.6\left(\mathrm{~d}, J_{\mathrm{C}-\mathrm{P}}=81.9 \mathrm{~Hz}\right), 109.1$, $121.8,125.2,126.2,127.1,127.2,127.8\left(\mathrm{~d}, J_{\mathrm{C}-\mathrm{P}}=11.5 \mathrm{~Hz}\right), 128.2(\mathrm{~d}$, $\left.J_{\text {C-P }}=11.4 \mathrm{~Hz}\right), 128.4,129.8,130.2\left(\mathrm{~d}, J_{\mathrm{C}-\mathrm{P}}=95.5 \mathrm{~Hz}\right), 130.4\left(\mathrm{~d}, J_{\mathrm{C}-\mathrm{P}}=\right.$ 95.7 Hz), 131.7, 132.4 (d, $\left.J_{C-P}=8.6 \mathrm{~Hz}\right), 135.6,143.2\left(\mathrm{~d}, J_{\mathrm{C}-\mathrm{P}}=5.6 \mathrm{~Hz}\right)$, 173.9 .

${ }^{31} \mathrm{P}$ NMR (162 MHz, DMSO- $\left.d_{6}\right): \delta=28.1$.

HRMS: $m / z[\mathrm{M}+\mathrm{H}]$ calcd. for $\mathrm{C}_{27} \mathrm{H}_{22} \mathrm{NO}_{3} \mathrm{P}+\mathrm{H}$; 440.1416; found: 440.1422 .

5-Chloro-3-(diphenyl-phosphinoyl)-3-hydroxy-indolin-2-one (3e) The title compound was prepared by following the general procedure for Scheme 1, using 5-chloroisatin 1 e $(0.5 \mathrm{mmol}, 0.091 \mathrm{~g})$ and diphenylphosphine oxide $\mathbf{2 a}(0.5 \mathrm{mmol}, 0.101 \mathrm{~g})$, providing $3 \mathbf{e}$ as a white solid. Yield: $0.180 \mathrm{~g}(94 \%) ; \mathrm{mp} 128^{\circ} \mathrm{C}$.

${ }^{1} \mathrm{H}$ NMR $\left(400 \mathrm{MHz}\right.$, DMSO- $\left.d_{6}\right): \delta=6.23(\mathrm{~s}, 1 \mathrm{H}), 6.77(\mathrm{~d}, J=8.4 \mathrm{~Hz}$, $1 \mathrm{H}), 7.25$ (d, $J=8.4 \mathrm{~Hz}, 1 \mathrm{H}), 7.45-7.54(\mathrm{~m}, 3 \mathrm{H}), 7.55-7.63(\mathrm{~m}, 3 \mathrm{H})$, $7.68(\mathrm{t}, J=7.2 \mathrm{~Hz}, 1 \mathrm{H}), 7.85-8.04(\mathrm{~m}, 4 \mathrm{H}), 10.65(\mathrm{~s}, 1 \mathrm{H})$.

${ }^{13} \mathrm{C}$ NMR $\left(100 \mathrm{MHz}\right.$, DMSO- $\left.d_{6}\right): \delta=79.9\left(\mathrm{~d}, J_{\mathrm{C}-\mathrm{P}}=80.5 \mathrm{~Hz}\right), 111.0,125.0$ $\left(\mathrm{d}, J_{\mathrm{C}-\mathrm{P}}=2.5 \mathrm{~Hz}\right), 125.5\left(\mathrm{~d}, J_{\mathrm{C}-\mathrm{P}}=2.8 \mathrm{~Hz}\right), 128.0\left(\mathrm{~d}, J_{\mathrm{C}-\mathrm{P}}=11.5 \mathrm{~Hz}\right), 128.3$ $\left(\mathrm{d}, J_{\mathrm{C}-\mathrm{P}}=11.3 \mathrm{~Hz}\right), 128.7,129.6,129.9\left(\mathrm{~d}, J_{\mathrm{C}-\mathrm{P}}=95.6 \mathrm{~Hz}\right), 130.1\left(\mathrm{~d}, J_{\mathrm{C}-\mathrm{P}}=\right.$ $92.8 \mathrm{~Hz}), 131.9,132.4\left(\mathrm{~d}, J_{\mathrm{C}-\mathrm{P}}=8.6 \mathrm{~Hz}\right), 132.5,141.7\left(\mathrm{~d}, J_{\mathrm{C}-\mathrm{P}}=6.1 \mathrm{~Hz}\right)$, 175.1 .

${ }^{31} \mathrm{P}$ NMR (162 MHz, $\mathrm{CDCl}_{3} /$ DMSO- $d_{6}$ ): $\delta=29.0$.

HRMS: $m / z[\mathrm{M}+\mathrm{H}]$ calcd. for $\mathrm{C}_{20} \mathrm{H}_{15} \mathrm{ClNO}_{3} \mathrm{P}+\mathrm{H}$ : 384.0556; found: 384.0540 .

\section{5-Chloro-3-(diphenyl-phosphinoyl)-3-hydroxy-1-methylindolin- 2-one (3f)}

The title compound was prepared by following the general procedure for Scheme 1, using 5-chloro-1-methylisatin 1f (0.5 mmol, $0.098 \mathrm{~g})$ and diphenylphosphine oxide $\mathbf{2 a}(0.5 \mathrm{mmol}, 0.101 \mathrm{~g})$, providing $3 \mathbf{f}$ as a white solid. Yield: $0.190 \mathrm{~g}(96 \%) ; \mathrm{mp} 136{ }^{\circ} \mathrm{C}$.

${ }^{1} \mathrm{H} \mathrm{NMR}\left(400 \mathrm{MHz}, \mathrm{CDCl}_{3}\right): \delta=2.87(\mathrm{~s}, 3 \mathrm{H}), 5.35$ (bs, $\left.1 \mathrm{H}\right), 6.53(\mathrm{~d}, J=$ $8.4 \mathrm{~Hz}, 1 \mathrm{H}), 6.94(\mathrm{~s}, 1 \mathrm{H}), 7.23(\mathrm{~d}, J=8.4 \mathrm{~Hz}, 1 \mathrm{H}), 7.37-7.43(\mathrm{~m}, 2 \mathrm{H})$, $7.48-7.51(\mathrm{~m}, 3 \mathrm{H}), 7.61(\mathrm{~d}, J=7.2 \mathrm{~Hz}, 1 \mathrm{H}), 7.72(\mathrm{~d}, J=8.0 \mathrm{~Hz}, 1 \mathrm{H})$, $7.75(\mathrm{~d}, J=8.0 \mathrm{~Hz}, 1 \mathrm{H}), 7.98(\mathrm{~d}, J=8.0 \mathrm{~Hz}, 1 \mathrm{H}), 8.01(\mathrm{~d}, J=8.0 \mathrm{~Hz}$, $1 \mathrm{H})$.

${ }^{13} \mathrm{C}$ NMR $\left(100 \mathrm{MHz}, \mathrm{CDCl}_{3}\right): \delta=26.4,80.5\left(\mathrm{~d}, J_{\mathrm{C}-\mathrm{P}}=70.4 \mathrm{~Hz}\right), 109.1$, $126.4,126.9\left(\mathrm{~d}, J_{\mathrm{C}-\mathrm{P}}=3.2 \mathrm{~Hz}\right), 127.4,127.5\left(\mathrm{~d}, J_{\mathrm{C}-\mathrm{P}}=96.9 \mathrm{~Hz}\right), 128.1$ (d, $\left.J_{\mathrm{C}-\mathrm{P}}=12.2 \mathrm{~Hz}\right), 128.5\left(\mathrm{~d}, J_{\mathrm{C}-\mathrm{P}}=12.1 \mathrm{~Hz}\right), 128.9,130.2,130.7,132.4(\mathrm{~d}$, $\left.J_{\text {C-P }}=9.2 \mathrm{~Hz}\right), 132.6\left(\mathrm{~d}, J_{\mathrm{C}-\mathrm{P}}=2.3 \mathrm{~Hz}\right), 132.8\left(\mathrm{~d}, J_{\mathrm{C}-\mathrm{P}}=2.5 \mathrm{~Hz}\right), 133.4(\mathrm{~d}$, $\left.J_{\text {C-P }}=9.0 \mathrm{~Hz}\right), 142.4,173.8$.

${ }^{31} \mathrm{P}$ NMR $\left(162 \mathrm{MHz}, \mathrm{CDCl}_{3}\right): \delta=29.5$.

HRMS: $m / z[\mathrm{M}+\mathrm{H}]$ calcd. for $\mathrm{C}_{21} \mathrm{H}_{17} \mathrm{ClNO}_{3} \mathrm{P}+\mathrm{H}$ : 398.0713; found: 398.0719.

\section{5-Chloro-3-(diphenyl-phosphinoyl)-3-hydroxy-1-ethylindolin-2- one (3g)}

The title compound was prepared by following the general procedure for Scheme 1, using 5-chloro-1-ethylisatin $1 \mathrm{~g}(0.5 \mathrm{mmol}, 0.105 \mathrm{~g})$ and diphenylphosphine oxide $2 \mathbf{a}(0.5 \mathrm{mmol}, 0.101 \mathrm{~g})$, providing $\mathbf{3 g}$ as a white solid. Yield: $0.201 \mathrm{~g}(98 \%) ; \mathrm{mp} 120^{\circ} \mathrm{C}$. 
${ }^{1} \mathrm{H}$ NMR $\left(400 \mathrm{MHz}, \mathrm{CDCl}_{3}\right): \delta=0.88(\mathrm{t}, J=7.2 \mathrm{~Hz}, 3 \mathrm{H}), 3.28-3.33(\mathrm{~m}$, $1 \mathrm{H}), 3.52-3.57(\mathrm{~m}, 1 \mathrm{H}), 5.53(\mathrm{~s}, 1 \mathrm{H}), 6.56(\mathrm{~d}, J=8.0 \mathrm{~Hz}, 1 \mathrm{H}), 7.02(\mathrm{~s}$, $1 \mathrm{H}), 7.24(\mathrm{~d}, J=8.0 \mathrm{~Hz}, 1 \mathrm{H}), 7.34-7.44(\mathrm{~m}, 2 \mathrm{H}), 7.45-7.55(\mathrm{~m}, 3 \mathrm{H})$, $7.61(\mathrm{t}, J=8.4 \mathrm{~Hz}, 1 \mathrm{H}), 7.68(\mathrm{~d}, J=7.6 \mathrm{~Hz}, 1 \mathrm{H}), 7.71(\mathrm{~d}, J=7.6 \mathrm{~Hz}, 1 \mathrm{H})$, $7.95(\mathrm{~d}, J=7.6 \mathrm{~Hz}, 1 \mathrm{H}), 7.98(\mathrm{~d}, J=7.6 \mathrm{~Hz}, 1 \mathrm{H})$.

${ }^{13} \mathrm{C}$ NMR $\left(100 \mathrm{MHz}, \mathrm{CDCl}_{3}\right): \delta=12.0,35.1,80.1\left(\mathrm{~d}, J_{\mathrm{C}-\mathrm{P}}=70.3 \mathrm{~Hz}\right)$, 109.0, 126.5, 126.8, 127.3, 127.6, 127.7, 128.0 (d, $\left.J_{\mathrm{C}-\mathrm{P}}=2.6 \mathrm{~Hz}\right), 128.2$ $\left(\mathrm{d}, J_{\mathrm{C}-\mathrm{P}}=11.9 \mathrm{~Hz}\right), 128.5\left(\mathrm{~d}, J_{\mathrm{C}-\mathrm{P}}=11.9 \mathrm{~Hz}\right), 130.2,132.4\left(\mathrm{~d}, J_{\mathrm{C}-\mathrm{P}}=\right.$ $9.3 \mathrm{~Hz}), 132.7\left(\mathrm{~d}, J_{\mathrm{C}-\mathrm{P}}=2.1 \mathrm{~Hz}\right), 132.8\left(\mathrm{~d}, J_{\mathrm{C}-\mathrm{P}}=2.4 \mathrm{~Hz}\right), 133.0\left(\mathrm{~d}, J_{\mathrm{C}-\mathrm{P}}=\right.$ $9.0 \mathrm{~Hz}), 141.7\left(\mathrm{~d}, J_{\mathrm{C}-\mathrm{P}}=5.8 \mathrm{~Hz}\right), 173.1$.

${ }^{31} \mathrm{P}$ NMR $\left(162 \mathrm{MHz}, \mathrm{CDCl}_{3}\right): \delta=30.9$.

HRMS: $m / z[\mathrm{M}+\mathrm{H}]$ calcd. for $\mathrm{C}_{22} \mathrm{H}_{19} \mathrm{ClNO}_{3} \mathrm{P}+\mathrm{H}$ : 412.0869; found: 412.0855 .

\section{5-Chloro-3-(diphenyl-phosphinoyl)-3-hydroxy-1-benzylindolin- 2-one (3h)}

The title compound was prepared by following the general procedure for Scheme 1, using 5-chloro-1-benzylisatin $\mathbf{1 h}(0.5 \mathrm{mmol}, 0.135 \mathrm{~g})$ and diphenylphosphine oxide $\mathbf{2 a}(0.5 \mathrm{mmol}, 0.101 \mathrm{~g})$, providing $\mathbf{3 h}$ as a white solid. Yield: $0.227 \mathrm{~g}(96 \%) ; \mathrm{mp} 138{ }^{\circ} \mathrm{C}$.

${ }^{1} \mathrm{H}$ NMR $\left(400 \mathrm{MHz}, \mathrm{CDCl}_{3}\right): \delta=4.69-4.78(\mathrm{~m}, 2 \mathrm{H}), 6.47(\mathrm{~d}, J=8.4 \mathrm{~Hz}$, $1 \mathrm{H}), 6.68$ (s, $1 \mathrm{H}), 7.09$ (d, $J=8.4 \mathrm{~Hz}, 1 \mathrm{H}), 7.18-7.31$ (m, $5 \mathrm{H}), 7.37-$ $7.45(\mathrm{~m}, 2 \mathrm{H}), 7.47-7.50(\mathrm{~m}, 3 \mathrm{H}), 7.63(\mathrm{t}, J=8.4 \mathrm{~Hz}, 1 \mathrm{H}), 7.95(\mathrm{~d}, J=$ $8.0 \mathrm{~Hz}, 1 \mathrm{H}), 7.98$ (d, J = 8.0 Hz, $1 \mathrm{H}), 8.10$ (d, $J=8.0 \mathrm{~Hz}, 1 \mathrm{H}), 8.13$ (d, $J=8.0 \mathrm{~Hz}, 1 \mathrm{H})$.

${ }^{13} \mathrm{C}$ NMR $\left(100 \mathrm{MHz}, \mathrm{CDCl}_{3} / \mathrm{DMSO}_{-} d_{6}\right): \delta=43.4,79.6\left(\mathrm{~d}, J_{\mathrm{C}-\mathrm{P}}=78.7 \mathrm{~Hz}\right)$, 109.4, 110.0, 125.8, 126.7, 126.9, 127.0, $127.4\left(\mathrm{~d}, J_{\mathrm{C}-\mathrm{P}}=12.3 \mathrm{~Hz}\right), 127.6$ $\left(\mathrm{d}, J_{\mathrm{C}-\mathrm{P}}=11.7 \mathrm{~Hz}\right), 128.0,128.2,128.4,129.1,130.2\left(\mathrm{~d}, J_{\mathrm{C}-\mathrm{P}}=91.2 \mathrm{~Hz}\right)$, 131.5, 131.9, $132.2\left(\mathrm{~d}, J_{\mathrm{C}-\mathrm{P}}=8.9 \mathrm{~Hz}\right), 132.4\left(\mathrm{~d}, J_{\mathrm{C}-\mathrm{P}}=8.6 \mathrm{~Hz}\right), 134.3$, $141.3\left(\mathrm{~d}, J_{\mathrm{C}-\mathrm{P}}=5.7 \mathrm{~Hz}\right), 173.6$

${ }^{31} \mathrm{P}$ NMR $\left(162 \mathrm{MHz}, \mathrm{CDCl}_{3} / \mathrm{DMSO} \mathrm{d}_{6}\right): \delta=28.1$.

HRMS: $m / z[\mathrm{M}+\mathrm{H}]$ calcd. for $\mathrm{C}_{27} \mathrm{H}_{21} \mathrm{ClNO}_{3} \mathrm{P}+\mathrm{H}$ : 474.1026; found: 474.1036 .

5-Bromo-3-(diphenyl-phosphinoyl)-3-hydroxy-indolin-2-one (3i) The title compound was prepared by following the general procedure for Scheme 1, using 5-bromoisatin $\mathbf{1 i}(0.5 \mathrm{mmol}, 0.113 \mathrm{~g})$ and diphenylphosphine oxide $\mathbf{2 a}(0.5 \mathrm{mmol}, 0.101 \mathrm{~g})$, providing $\mathbf{3 i}$ as a white solid. Yield: $0.194 \mathrm{~g} \mathrm{(91 \% );} \mathrm{mp} 158^{\circ} \mathrm{C}$.

${ }^{1} \mathrm{H}$ NMR $\left(400 \mathrm{MHz}, \mathrm{CDCl}_{3} /\right.$ DMSO- $\left._{6}\right): \delta=6.58(\mathrm{~s}, 1 \mathrm{H}), 6.67(\mathrm{~d}, J=$ $8.4 \mathrm{~Hz}, 1 \mathrm{H}), 7.10-7.15(\mathrm{~m}, 1 \mathrm{H}), 7.27(\mathrm{~d}, J=8.0 \mathrm{~Hz}, 1 \mathrm{H}), 7.37-7.70(\mathrm{~m}$, $6 \mathrm{H}), 7.90-8.10$ (m, $4 \mathrm{H}), 10.34$ (s, $1 \mathrm{H})$.

${ }^{13} \mathrm{C}$ NMR $\left(100 \mathrm{MHz}, \mathrm{CDCl}_{3} / \mathrm{DMSO}_{-} d_{6}\right): \delta=79.3\left(\mathrm{~d}, J_{\mathrm{C}-\mathrm{P}}=79.3 \mathrm{~Hz}\right)$, $110.3,112.4,126.8\left(\mathrm{~d}, J_{\mathrm{C}-\mathrm{P}}=11.9 \mathrm{~Hz}\right), 127.0\left(\mathrm{~d}, J_{\mathrm{C}-\mathrm{P}}=11.7 \mathrm{~Hz}\right), 127.8$, $128.0\left(\mathrm{~d}, J_{\mathrm{C}-\mathrm{P}}=2.5 \mathrm{~Hz}\right), 128.4\left(\mathrm{~d}, J_{\mathrm{C}-\mathrm{P}}=96.5 \mathrm{~Hz}\right), 128.7\left(\mathrm{~d}, J_{\mathrm{C}-\mathrm{P}}=\right.$ $96.3 \mathrm{~Hz}), 130.8\left(\mathrm{~d}, J_{\mathrm{C}-\mathrm{P}}=2.1 \mathrm{~Hz}\right), 131.3,131.6\left(\mathrm{~d}, J_{\mathrm{C}-\mathrm{P}}=9.2 \mathrm{~Hz}\right), 131.8$ $\left(\mathrm{d}, J_{\mathrm{C}-\mathrm{P}}=8.9 \mathrm{~Hz}\right), 140.9\left(\mathrm{~d}, J_{\mathrm{C}-\mathrm{P}}=6.0 \mathrm{~Hz}\right), 174.4$.

${ }^{31} \mathrm{P}$ NMR $\left(162 \mathrm{MHz}, \mathrm{CDCl}_{3} / \mathrm{DMSO} \mathrm{d}_{6}\right): \delta=28.3$.

HRMS: $m / z[\mathrm{M}+\mathrm{H}]$ calcd. for $\mathrm{C}_{20} \mathrm{H}_{15} \mathrm{BrNO}_{3} \mathrm{P}+\mathrm{H}$ : 428.0051; found: 428.0041 .

\section{5-Bromo-3-(diphenyl-phosphinoyl)-3-hydroxy-1-ethylindolin-2- one ( $3 \mathbf{j})$}

The title compound was prepared by following the general procedure for Scheme 1, using 5-bromo-1-ethylisatin $\mathbf{1 j}(0.5 \mathrm{mmol}, 0.127 \mathrm{~g})$ and diphenylphosphine oxide $\mathbf{2 a}(0.5 \mathrm{mmol}, 0.101 \mathrm{~g})$, providing $\mathbf{3 j}$ as a white solid. Yield: $0.216 \mathrm{~g}(95 \%) ; \mathrm{mp} 132{ }^{\circ} \mathrm{C}$.
${ }^{1} \mathrm{H}$ NMR (400 MHz, DMSO- $\left.d_{6}\right): \delta=0.93(\mathrm{t}, J=7.2 \mathrm{~Hz}, 3 \mathrm{H}$ ), 3.49-3.56 $(\mathrm{m}, 2 \mathrm{H}), 6.60(\mathrm{~s}, 1 \mathrm{H}), 6.96(\mathrm{~d}, J=8.4 \mathrm{~Hz}, 1 \mathrm{H}), 7.42-7.62(\mathrm{~m}, 7 \mathrm{H}), 7.69$ $(\mathrm{t}, J=7.2 \mathrm{~Hz}, 1 \mathrm{H}), 7.76(\mathrm{~d}, J=7.6 \mathrm{~Hz}, 1 \mathrm{H}), 7.79(\mathrm{~d}, J=7.2 \mathrm{~Hz}, 1 \mathrm{H}), 7.98$ $(\mathrm{d}, J=8.0 \mathrm{~Hz}, 1 \mathrm{H}), 8.01(\mathrm{~d}, J=7.2 \mathrm{~Hz}, 1 \mathrm{H})$.

${ }^{13} \mathrm{C}$ NMR $\left(100 \mathrm{MHz}\right.$, DMSO- $\left.d_{6}\right): \delta=12.0,34.4,79.6\left(\mathrm{~d}, J_{\mathrm{C}-\mathrm{P}}=79.3 \mathrm{~Hz}\right)$, 110.5, 113.3, 128.0 (d, $\left.J_{C-P}=11.5 \mathrm{~Hz}\right), 128.3$ (d, $J_{C-P}=11.0 \mathrm{~Hz}$ ), 128.5 $\left(\mathrm{d}, J_{\mathrm{C}-\mathrm{P}}=2.4 \mathrm{~Hz}\right), 129.6\left(\mathrm{~d}, J_{\mathrm{C}-\mathrm{P}}=96.3 \mathrm{~Hz}\right), 129.8\left(\mathrm{~d}, J_{\mathrm{C}-\mathrm{p}}=95.6 \mathrm{~Hz}\right)$, 132.0, $132.1\left(\mathrm{~d}, J_{\mathrm{C}-\mathrm{P}}=8.9 \mathrm{~Hz}\right), 132.5\left(\mathrm{~d}, J_{\mathrm{C}-\mathrm{P}}=8.7 \mathrm{~Hz}\right), 142.3\left(\mathrm{~d}, J_{\mathrm{C}-\mathrm{P}}=\right.$ $5.5 \mathrm{~Hz}), 172.9$.

${ }^{31} \mathrm{P}$ NMR $\left(162 \mathrm{MHz}, \mathrm{DMSO}-d_{6}\right): \delta=28.1$.

HRMS: $m / z[\mathrm{M}+\mathrm{H}]$ calcd. for $\mathrm{C}_{22} \mathrm{H}_{19} \mathrm{BrNO}_{3} \mathrm{P}+\mathrm{H}$ : 456.0364; found: 456.0370 .

\section{3-(Diphenyl-phosphinoyl)-3-hydroxy-5-nitro-indolin-2-one (3k)} The title compound was prepared by following the general procedure for Scheme 1, using 5-nitroisatin $1 \mathbf{k}(0.5 \mathrm{mmol}, 0.096 \mathrm{~g})$ and diphenylphosphine oxide $2 \mathbf{a}(0.5 \mathrm{mmol}, 0.101 \mathrm{~g})$, providing $3 \mathbf{k}$ as a white solid. Yield: $0.181 \mathrm{~g}(92 \%) ; \mathrm{mp} 142{ }^{\circ} \mathrm{C}$.

${ }^{1} \mathrm{H}$ NMR $\left(400 \mathrm{MHz}\right.$, DMSO- $\left.d_{6}\right): \delta=6.97(\mathrm{~d}, J=8.8 \mathrm{~Hz}, 1 \mathrm{H}), 7.09(\mathrm{~s}$, $1 \mathrm{H}), 7.48-7.52(\mathrm{~m}, 2 \mathrm{H}), 7.55-7.75(\mathrm{~m}, 5 \mathrm{H}), 7.90-8.00(\mathrm{~m}, 4 \mathrm{H}), 8.18$ (dd, $J=8.8,1.2 \mathrm{~Hz}, 1 \mathrm{H}), 11.2(\mathrm{~s}, 1 \mathrm{H})$.

${ }^{13} \mathrm{C}$ NMR $\left(100 \mathrm{MHz}\right.$, DMSO- $\left.d_{6}\right): \delta=79.7\left(\mathrm{~d}, J_{\mathrm{C}-\mathrm{p}}=79.2 \mathrm{~Hz}\right), 109.8$, $120.8,127.0,127.7,128.1$ (d, $\left.J_{\text {C-P }}=11.5 \mathrm{~Hz}\right), 128.4\left(\mathrm{~d}, J_{\mathrm{C}-\mathrm{P}}=11.5 \mathrm{~Hz}\right.$ ), $129.3\left(\mathrm{~d}, J_{\mathrm{C}-\mathrm{P}}=96.1 \mathrm{~Hz}\right), 129.5\left(\mathrm{~d}, J_{\mathrm{C}-\mathrm{P}}=96.4 \mathrm{~Hz}\right), 132.1,132.3\left(\mathrm{~d}, J_{\mathrm{C}-\mathrm{P}}=\right.$ $2.0 \mathrm{~Hz}), 132.4\left(\mathrm{~d}, J_{\mathrm{C}-\mathrm{P}}=2.5 \mathrm{~Hz}\right), 132.7,141.4,149.1$ (d, $\left.J_{\mathrm{C}-\mathrm{P}}=5.5 \mathrm{~Hz}\right)$, 175.8 .

${ }^{31} \mathrm{P}$ NMR $\left(162 \mathrm{MHz}\right.$, DMSO- $\left.d_{6}\right): \delta=27.7$

HRMS: $m / z[\mathrm{M}+\mathrm{H}]$ calcd. for $\mathrm{C}_{20} \mathrm{H}_{15} \mathrm{~N}_{2} \mathrm{O}_{5} \mathrm{P}+\mathrm{H}$ : 395.0797; found: 395.0785 .

3-(Di(4-methylphenyl)phosphinoyl)-3-hydroxy-indolin-2-one (31) The title compound was prepared by following the general procedure for Scheme 1, using isatin $\mathbf{1 a}(0.5 \mathrm{mmol}, 0.073 \mathrm{~g})$ and di ( $p$-tolyl $)$ phosphine oxide $\mathbf{2 b}(0.5 \mathrm{mmol}, 0.115 \mathrm{~g})$, providing 31 as a white solid. Yield: $0.175 \mathrm{~g}(93 \%) ; \mathrm{mp} 152{ }^{\circ} \mathrm{C}$.

${ }^{1} \mathrm{H}$ NMR $\left(400 \mathrm{MHz}\right.$, DMSO- $\left.d_{6}\right): \delta=2.32(\mathrm{~s}, 3 \mathrm{H}), 2.38(\mathrm{~s}, 3 \mathrm{H}), 6.44(\mathrm{~d}$, $J=7.6 \mathrm{~Hz}, 1 \mathrm{H}), 6.71-6.77(\mathrm{~m}, 2 \mathrm{H}), 7.11-7.29(\mathrm{~m}, 4 \mathrm{H}), 7.34(\mathrm{~s}, 1 \mathrm{H})$, 7.36 (s, $1 \mathrm{H}), 7.66-7.91(\mathrm{~m}, 4 \mathrm{H}), 10.4(\mathrm{~s}, 1 \mathrm{H})$.

${ }^{13} \mathrm{C}$ NMR $\left(100 \mathrm{MHz}\right.$, DMSO- $\left.d_{6}\right): \delta=21.1,21.2,79.8\left(\mathrm{~d}, J_{\mathrm{C}-\mathrm{P}}=81.8 \mathrm{~Hz}\right)$, 109.4, 121.0, 125.6, 127.0, $127.4\left(\mathrm{~d}, J_{\mathrm{C}-\mathrm{P}}=97.7 \mathrm{~Hz}\right), 127.6\left(\mathrm{~d}, J_{\mathrm{C}-\mathrm{P}}=\right.$ $97.7 \mathrm{~Hz}), 128.4\left(\mathrm{~d}, J_{\mathrm{C}-\mathrm{P}}=11.8 \mathrm{~Hz}\right), 128.7\left(\mathrm{~d}, J_{\mathrm{C}-\mathrm{P}}=11.7 \mathrm{~Hz}\right), 129.7,132.3$ $\left(\mathrm{d}, J_{\mathrm{C}-\mathrm{P}}=9.0 \mathrm{~Hz}\right), 141.5,142.0,142.8\left(\mathrm{~d}, J_{\mathrm{C}-\mathrm{P}}=5.8 \mathrm{~Hz}\right), 175.4$.

${ }^{31} \mathrm{P}$ NMR $\left(162 \mathrm{MHz}, \mathrm{DMSO}-d_{6}\right): \delta=27.9$.

HRMS: $m / z[\mathrm{M}+\mathrm{H}]$ calcd. for $\mathrm{C}_{22} \mathrm{H}_{20} \mathrm{NO}_{3} \mathrm{P}+\mathrm{H}$ : 378.1259; found: 378.1265 .

\section{3-(Di(4-methylphenyl)phosphinoyl)-3-hydroxy-1-ethylindolin-2-} one $(3 \mathrm{~m})$

The title compound was prepared by following the general procedure for Scheme 1, using 1-ethylisatin 1c $(0.5 \mathrm{mmol}, 0.087 \mathrm{~g})$ and $\mathrm{di}(p$ tolyl)phosphine oxide $\mathbf{2 b}(0.5 \mathrm{mmol}, 0.115 \mathrm{~g})$, providing $\mathbf{3 m}$ as a white solid. Yield: $0.194 \mathrm{~g}(96 \%) ; \mathrm{mp} 140{ }^{\circ} \mathrm{C}$.

${ }^{1} \mathrm{H}$ NMR $\left(400 \mathrm{MHz}, \mathrm{CDCl}_{3}\right): \delta=0.87(\mathrm{t}, J=7.2 \mathrm{~Hz}, 3 \mathrm{H}), 2.36(\mathrm{~s}, 3 \mathrm{H})$, $2.41(\mathrm{~s}, 3 \mathrm{H}), 3.32-3.37(\mathrm{~m}, 1 \mathrm{H}), 3.57-3.63(\mathrm{~m}, 1 \mathrm{H}), 4.58(\mathrm{bs}, 1 \mathrm{H})$, $6.66(\mathrm{~d}, J=8.0 \mathrm{~Hz}, 1 \mathrm{H}), 6.89(\mathrm{t}, J=7.6 \mathrm{~Hz}, 1 \mathrm{H}), 6.98(\mathrm{~d}, J=7.6 \mathrm{~Hz}, 1 \mathrm{H})$, $7.170(\mathrm{~d}, J=8.0 \mathrm{~Hz}, 1 \mathrm{H}), 7.176(\mathrm{~d}, J=8.0 \mathrm{~Hz}, 1 \mathrm{H}), 7.26-7.29(\mathrm{~m}, 3 \mathrm{H})$, $7.57(\mathrm{~d}, J=8.0 \mathrm{~Hz}, 1 \mathrm{H}), 7.60(\mathrm{~d}, J=8.0 \mathrm{~Hz}, 1 \mathrm{H}), 7.75(\mathrm{~d}, J=8.0 \mathrm{~Hz}$, $1 \mathrm{H}), 7.78(\mathrm{~d}, J=8.0 \mathrm{~Hz}, 1 \mathrm{H})$. 
${ }^{13} \mathrm{C}$ NMR $\left(100 \mathrm{MHz}, \mathrm{CDCl}_{3}\right): \delta=12.0,21.7,34.9,79.8\left(\mathrm{~d}, J_{\mathrm{C}-\mathrm{P}}=70.8 \mathrm{~Hz}\right)$, 108.2, 122.6, $124.0\left(\mathrm{~d}, J_{\mathrm{C}-\mathrm{P}}=98.4 \mathrm{~Hz}\right), 124.5,125.4\left(\mathrm{~d}, J_{\mathrm{C}-\mathrm{P}}=99.9 \mathrm{~Hz}\right)$, $126.8\left(\mathrm{~d}, J_{\mathrm{C}-\mathrm{P}}=3.0 \mathrm{~Hz}\right), 128.9\left(\mathrm{~d}, J_{\mathrm{C}-\mathrm{P}}=12.3 \mathrm{~Hz}\right), 129.1\left(\mathrm{~d}, J_{\mathrm{C}-\mathrm{P}}=\right.$ $11.9 \mathrm{~Hz}), 130.3,132.4\left(\mathrm{~d}, J_{\mathrm{C}-\mathrm{P}}=9.5 \mathrm{~Hz}\right), 132.9\left(\mathrm{~d}, J_{\mathrm{C}-\mathrm{P}}=8.9 \mathrm{~Hz}\right), 143.0$ $\left(\mathrm{d}, J_{\mathrm{C}-\mathrm{P}}=2.4 \mathrm{~Hz}\right), 143.2\left(\mathrm{~d}, J_{\mathrm{C}-\mathrm{P}}=2.7 \mathrm{~Hz}\right), 143.5\left(\mathrm{~d}, J_{\mathrm{C}-\mathrm{P}}=5.4 \mathrm{~Hz}\right), 173.6$. ${ }^{31} \mathrm{P}$ NMR $\left(162 \mathrm{MHz}, \mathrm{CDCl}_{3}\right): \delta=30.9$.

HRMS: $m / z[\mathrm{M}+\mathrm{H}]$ calcd. for $\mathrm{C}_{24} \mathrm{H}_{24} \mathrm{NO}_{3} \mathrm{P}+\mathrm{H}$ : 406.1572; found: 406.1574 .

\section{5-Chloro-3-(di(4-methylphenyl)phosphinoyl)-3-hydroxy-indolin- 2-one (3n)}

The title compound was prepared by following the general procedure for Scheme 1, using 5-chloroisatin $\mathbf{1 e}(0.5 \mathrm{mmol}, 0.090 \mathrm{~g})$ and di $(p-$ tolyl)phosphine oxide $\mathbf{2 b}(0.5 \mathrm{mmol}, 0.115 \mathrm{~g})$, providing $\mathbf{3 n}$ as a white solid. Yield: $0.185 \mathrm{~g}(90 \%) ; \mathrm{mp} 138^{\circ} \mathrm{C}$.

${ }^{1} \mathrm{H}$ NMR $\left(400 \mathrm{MHz}\right.$, DMSO- $\left.d_{6}\right): \delta=2.32(\mathrm{~s}, 3 \mathrm{H}), 2.39(\mathrm{~s}, 3 \mathrm{H}), 6.33(\mathrm{~s}$, $1 \mathrm{H}), 6.75(\mathrm{~d}, J=8.4 \mathrm{~Hz}, 1 \mathrm{H}), 7.23-7.42(\mathrm{~m}, 6 \mathrm{H}), 7.73(\mathrm{~d}, J=8.0 \mathrm{~Hz}$, $1 \mathrm{H}), 7.75(\mathrm{~d}, J=8.0 \mathrm{~Hz}, 1 \mathrm{H}), 7.81(\mathrm{~d}, J=8.4 \mathrm{~Hz}, 1 \mathrm{H}), 7.84(\mathrm{~d}, J=$ $8.4 \mathrm{~Hz}, 1 \mathrm{H}), 10.6(\mathrm{~s}, 1 \mathrm{H})$

${ }^{13} \mathrm{C}$ NMR $\left(100 \mathrm{MHz}\right.$, DMSO- $\left.d_{6}\right): \delta=21.1,22.2,80.0\left(\mathrm{~d}, J_{\mathrm{C}-\mathrm{p}}=80.2 \mathrm{~Hz}\right)$, $110.8,124.9\left(\mathrm{~d}, J_{\mathrm{C}-\mathrm{P}}=2.4 \mathrm{~Hz}\right), 125.6,126.8\left(\mathrm{~d}, J_{\mathrm{C}-\mathrm{P}}=98.3 \mathrm{~Hz}\right), 127.0(\mathrm{~d}$, $\left.J_{C-P}=98.5 \mathrm{~Hz}\right), 128.5\left(\mathrm{~d}, J_{\mathrm{C}-\mathrm{P}}=11.8 \mathrm{~Hz}\right), 128.8\left(\mathrm{~d}, J_{\mathrm{C}-\mathrm{P}}=11.9 \mathrm{~Hz}\right), 128.9$, $129.5,132.3\left(\mathrm{~d}, J_{\mathrm{C}-\mathrm{P}}=9.2 \mathrm{~Hz}\right), 132.5\left(\mathrm{~d}, J_{\mathrm{C}-\mathrm{P}}=9.1 \mathrm{~Hz}\right), 141.6\left(\mathrm{~d}, J_{\mathrm{C}-\mathrm{P}}=\right.$ $5.2 \mathrm{~Hz}), 141.8,142.4,175.2$.

${ }^{31}$ P NMR (162 MHz, DMSO- $\left.d_{6}\right): \delta=28.3$.

HRMS: $m / z[\mathrm{M}+\mathrm{H}]$ calcd. for $\mathrm{C}_{22} \mathrm{H}_{19} \mathrm{ClNO}_{3} \mathrm{P}+\mathrm{H}$ : 412.0869; found: 412.0875 .

\section{5-Chloro-3-(di(4-methylphenyl)phosphinoyl)-3-hydroxy-1- ethylindolin-2-one (3o)}

The title compound was prepared by following the general procedure for Scheme 1, using 5-chloro-1-ethylisatin $1 \mathrm{~g}(0.5 \mathrm{mmol}, 0.105 \mathrm{~g})$ and di( $p$-tolyl)phosphine oxide $\mathbf{2 b}(0.5 \mathrm{mmol}, 0.115 \mathrm{~g})$, providing $3 \mathbf{3 o}$ as a white solid. Yield: $0.206 \mathrm{~g}(94 \%) ; \mathrm{mp} 122^{\circ} \mathrm{C}$.

${ }^{1} \mathrm{H}$ NMR (400 MHz, $\left.\mathrm{CDCl}_{3}\right): \delta=0.86(\mathrm{t}, J=7.2 \mathrm{~Hz}, 3 \mathrm{H}), 2.34(\mathrm{~s}, 3 \mathrm{H})$, $2.42(\mathrm{~s}, 3 \mathrm{H}), 3.26-3.32(\mathrm{~m}, 1 \mathrm{H}), 3.49-3.54(\mathrm{~m}, 1 \mathrm{H}), 6.13$ (bs, $1 \mathrm{H})$, $6.51(\mathrm{~d}, J=8.4 \mathrm{~Hz}, 1 \mathrm{H}), 7.10-7.16(\mathrm{~m}, 3 \mathrm{H}), 7.21(\mathrm{~d}, J=8.0 \mathrm{~Hz}, 1 \mathrm{H})$, 7.23-7.30 (m, $2 \mathrm{H}), 7.51(\mathrm{~d}, J=8.0 \mathrm{~Hz}, 1 \mathrm{H}), 7.54(\mathrm{~d}, J=8.0 \mathrm{~Hz}, 1 \mathrm{H})$, $7.83(\mathrm{~d}, J=8.0 \mathrm{~Hz}, 1 \mathrm{H}), 7.86(\mathrm{~d}, J=8.4 \mathrm{~Hz}, 1 \mathrm{H})$.

${ }^{13} \mathrm{C}$ NMR $\left(100 \mathrm{MHz}, \mathrm{CDCl}_{3}\right): \delta=11.9,21.6,21.7,35.0,80.3\left(\mathrm{~d}, J_{\mathrm{C}-\mathrm{p}}=\right.$ $70.8 \mathrm{~Hz}$ ), 108.8, 124.2 (d, $\left.J_{\mathrm{C}-\mathrm{P}}=99.8 \mathrm{~Hz}\right), 124.9\left(\mathrm{~d}, J_{\mathrm{C}-\mathrm{P}}=99.9 \mathrm{~Hz}\right)$, $127.0,127.4\left(\mathrm{~d}, J_{\mathrm{C}-\mathrm{P}}=2.6 \mathrm{~Hz}\right), 127.9\left(\mathrm{~d}, J_{\mathrm{C}-\mathrm{P}}=2.6 \mathrm{~Hz}\right), 128.8\left(\mathrm{~d}, J_{\mathrm{C}-\mathrm{P}}=\right.$ $12.3 \mathrm{~Hz}), 129.1\left(\mathrm{~d}, J_{\mathrm{C}-\mathrm{P}}=12.4 \mathrm{~Hz}\right), 129.9,132.4\left(\mathrm{~d}, J_{\mathrm{C}-\mathrm{P}}=9.6 \mathrm{~Hz}\right), 133.0$ $\left(\mathrm{d}, J_{\mathrm{C}-\mathrm{P}}=9.5 \mathrm{~Hz}\right), 141.6\left(\mathrm{~d}, J_{\mathrm{C}-\mathrm{P}}=5.7 \mathrm{~Hz}\right), 143.0\left(\mathrm{~d}, J_{\mathrm{C}-\mathrm{P}}=2.6 \mathrm{~Hz}\right), 143.2$ $\left(\mathrm{d}, J_{\mathrm{C}-\mathrm{P}}=2.4 \mathrm{~Hz}\right), 173.2\left(\mathrm{~d}, J_{\mathrm{C}-\mathrm{P}}=5.2 \mathrm{~Hz}\right)$.

${ }^{31} \mathrm{P} \operatorname{NMR}\left(162 \mathrm{MHz}, \mathrm{CDCl}_{3}\right): \delta=31.8$.

HRMS: $m / z[\mathrm{M}+\mathrm{H}]$ calcd. for $\mathrm{C}_{24} \mathrm{H}_{23} \mathrm{ClNO}_{3} \mathrm{P}+\mathrm{H}$ : 440.1182 ; found: 440.1195 .

\section{3-(Bis(3,5-dimethylphenyl)phosphinoyl)-3-hydroxy-indolin-2- one (3p)}

The title compound was prepared following the general procedure for Scheme 1, using isatin 1a $(0.5 \mathrm{mmol}, 0.073 \mathrm{~g})$ and bis (3,5-dimethylphenyl)phosphine oxide $2 \mathrm{c}(0.5 \mathrm{mmol}, 0.129 \mathrm{~g})$, providing $3 \mathrm{p}$ as a white solid. Yield: $0.182 \mathrm{~g}(90 \%)$; $\mathrm{mp} 178{ }^{\circ} \mathrm{C}$.
${ }^{1} \mathrm{H}$ NMR $\left(400 \mathrm{MHz}, \mathrm{CDCl}_{3}\right): \delta=2.29(\mathrm{~s}, 6 \mathrm{H}), 2.31(\mathrm{~s}, 6 \mathrm{H}), 5.31(\mathrm{~d}, J=$ $6.4 \mathrm{~Hz}, 1 \mathrm{H}), 6.65(\mathrm{~d}, J=7.2 \mathrm{~Hz}, 1 \mathrm{H}), 6.69(\mathrm{~d}, J=7.6 \mathrm{~Hz}, 1 \mathrm{H}), 7.79(\mathrm{t}, J=$ 7.6 Hz, $1 \mathrm{H}), 7.11-7.23(\mathrm{~m}, 3 \mathrm{H}), 7.44(\mathrm{~s}, 1 \mathrm{H}), 7.46(\mathrm{~s}, 1 \mathrm{H}), 7.48(\mathrm{~s}$, $1 \mathrm{H}), 7.51(\mathrm{~s}, 1 \mathrm{H}), 9.31(\mathrm{~s}, 1 \mathrm{H})$.

${ }^{13} \mathrm{C}$ NMR $\left(100 \mathrm{MHz}, \mathrm{CDCl}_{3} / \mathrm{DMSO}_{-} d_{6}\right): \delta=21.3,80.2\left(\mathrm{~d}, J_{\mathrm{C}-\mathrm{P}}=72.7 \mathrm{~Hz}\right)$, $110.2,122.0,125.3,126.3$ (d, $\left.J_{\text {C-P }}=2.3 \mathrm{~Hz}\right), 127.4\left(\mathrm{~d}, J_{\mathrm{C}-\mathrm{P}}=91.9 \mathrm{~Hz}\right)$, $128.1,128.4\left(\mathrm{~d}, J_{\mathrm{C}-\mathrm{P}}=95.6 \mathrm{~Hz}\right), 130.1\left(\mathrm{~d}, J_{\mathrm{C}-\mathrm{P}}=8.5 \mathrm{~Hz}\right), 130.3\left(\mathrm{~d}, J_{\mathrm{C}-\mathrm{P}}=\right.$ $9.2 \mathrm{~Hz}), 134.1\left(\mathrm{~d}, J_{\mathrm{C}-\mathrm{P}}=2.4 \mathrm{~Hz}\right), 134.2\left(\mathrm{~d}, J_{\mathrm{C}-\mathrm{P}}=2.5 \mathrm{~Hz}\right), 137.6\left(\mathrm{~d}, J_{\mathrm{C}-\mathrm{P}}=\right.$ $12.6 \mathrm{~Hz}), 137.8\left(\mathrm{~d}, J_{\mathrm{C}-\mathrm{P}}=12.3 \mathrm{~Hz}\right), 142.4\left(\mathrm{~d}, J_{\mathrm{C}-\mathrm{P}}=6.1 \mathrm{~Hz}\right), 175.9$.

${ }^{31} \mathrm{P}$ NMR $\left(162 \mathrm{MHz}, \mathrm{CDCl}_{3} / \mathrm{DMSO}^{-d_{6}}\right): \delta=30.8$.

HRMS: $m / z[\mathrm{M}+\mathrm{H}]$ calcd. for $\mathrm{C}_{24} \mathrm{H}_{24} \mathrm{NO}_{3} \mathrm{P}+\mathrm{H}$ : 406.1572; found: 406.1582.

\section{3-(Bis(3,5-dimethylphenyl)phosphinoyl)-3-hydroxy-1-methylin- dolin-2-one (3q)}

The title compound was prepared by following the general procedure for Scheme 1, using 1-methylisatin $\mathbf{1 b}(0.5 \mathrm{mmol}, 0.080 \mathrm{~g})$ and bis (3,5-dimethylphenyl)phosphine oxide $2 \mathrm{c}(0.5 \mathrm{mmol}, 0.129 \mathrm{~g})$, providing $3 q$ as a white solid. Yield: $0.192 \mathrm{~g}(92 \%) ; \mathrm{mp} 138{ }^{\circ} \mathrm{C}$.

${ }^{1} \mathrm{H} \mathrm{NMR}\left(400 \mathrm{MHz}, \mathrm{CDCl}_{3} / \mathrm{DMSO}_{-} \mathrm{d}_{6}\right): \delta=2.27(\mathrm{~s}, 6 \mathrm{H}), 2.34(\mathrm{~s}, 6 \mathrm{H})$, $2.94(\mathrm{~s}, 3 \mathrm{H}), 6.65(\mathrm{~d}, J=8.0 \mathrm{~Hz}, 1 \mathrm{H}), 6.75(\mathrm{bs}, 1 \mathrm{H}), 6.85-6.95(\mathrm{~m}, 2 \mathrm{H})$, $7.10(\mathrm{~s}, 1 \mathrm{H}), 7.19(\mathrm{~s}, 1 \mathrm{H}), 7.26(\mathrm{t}, J=7.6 \mathrm{~Hz}, 1 \mathrm{H}), 7.36(\mathrm{~s}, 1 \mathrm{H}), 7.39(\mathrm{~s}$, $1 \mathrm{H}), 7.57(\mathrm{~s}, 1 \mathrm{H}), 7.60(\mathrm{~s}, 1 \mathrm{H})$.

${ }^{13} \mathrm{C}$ NMR $\left(100 \mathrm{MHz}, \mathrm{CDCl}_{3} / \mathrm{DMSO}_{-} \mathrm{d}_{6}\right): \delta=20.7,20.8,25.6,80.0(\mathrm{~d}$, $\left.J_{C-P}=75.5 \mathrm{~Hz}\right), 107.2,121.7,125.1,125.8,128.0\left(\mathrm{~d}, J_{\mathrm{C}-\mathrm{P}}=95.6 \mathrm{~Hz}\right)$, $128.4\left(\mathrm{~d}, J_{\mathrm{C}-\mathrm{P}}=95.6 \mathrm{~Hz}\right), 129.4\left(\mathrm{~d}, J_{\mathrm{C}-\mathrm{P}}=8.7 \mathrm{~Hz}\right), 129.8\left(\mathrm{~d}, J_{\mathrm{C}-\mathrm{P}}=8.9 \mathrm{~Hz}\right)$, $133.1,133.3,136.7\left(\mathrm{~d}, J_{\mathrm{C}-\mathrm{P}}=12.5 \mathrm{~Hz}\right), 137.0$ (d, $\left.J_{\mathrm{C}-\mathrm{P}}=12.4 \mathrm{~Hz}\right), 143.4$ $\left(\mathrm{d}, J_{\mathrm{C}-\mathrm{P}}=5.7 \mathrm{~Hz}\right), 173.5$.

${ }^{31} \mathrm{P}$ NMR $\left(162 \mathrm{MHz}, \mathrm{CDCl}_{3} / \mathrm{DMSO} \mathrm{d}_{6}\right): \delta=30.4$.

HRMS: $m / z[M+H]$ calcd. for $\mathrm{C}_{25} \mathrm{H}_{26} \mathrm{NO}_{3} \mathrm{P}+\mathrm{H}$ : 420.1729; found: 420.1737 .

\section{3-(Bis(3,5-dimethylphenyl)phosphinoyl)-3-hydroxy-1-ethylindo-} lin-2-one (3r)

The title compound was prepared by following the general procedure for Scheme 1, using 1-ethylisatin 1c $(0.5 \mathrm{mmol}, 0.087 \mathrm{~g})$ and bis $(3,5-$ dimethylphenyl)phosphine oxide $2 \mathbf{c}(0.5 \mathrm{mmol}, 0.129 \mathrm{~g})$, providing $3 \mathbf{r}$ as a white solid. Yield: $0.203 \mathrm{~g}(94 \%) ; \mathrm{mp} 132{ }^{\circ} \mathrm{C}$.

${ }^{1} \mathrm{H}$ NMR $\left(400 \mathrm{MHz}, \mathrm{CDCl}_{3}\right): \delta=0.83(\mathrm{t}, J=7.2 \mathrm{~Hz}, 3 \mathrm{H}), 2.26(\mathrm{~s}, 6 \mathrm{H})$, $2.31(\mathrm{~s}, 6 \mathrm{H}), 3.29-3.35(\mathrm{~m}, 1 \mathrm{H}), 3.51-3.62(\mathrm{~m}, 1 \mathrm{H}), 4.57(\mathrm{~s}, 1 \mathrm{H}), 6.66$ $(\mathrm{d}, J=7.6 \mathrm{~Hz}, 1 \mathrm{H}), 6.91(\mathrm{t}, J=7.6 \mathrm{~Hz}, 1 \mathrm{H}), 6.99(\mathrm{~d}, J=7.2 \mathrm{~Hz}, 1 \mathrm{H}), 7.12$ (s, $1 \mathrm{H}), 7.19$ (s, $1 \mathrm{H}), 7.24-7.34(\mathrm{~m}, 3 \mathrm{H}), 7.39$ (s, $1 \mathrm{H}), 7.42(\mathrm{~s}, 1 \mathrm{H})$.

${ }^{13} \mathrm{C}$ NMR $\left(100 \mathrm{MHz}, \mathrm{CDCl}_{3}\right): \delta=12.0,21.36,21.39,34.8,79.8\left(\mathrm{~d}, J_{\mathrm{C}-\mathrm{P}}=\right.$ $69.4 \mathrm{~Hz}), 107.9,122.5,124.4,126.8\left(\mathrm{~d}, J_{\mathrm{C}-\mathrm{P}}=95.7 \mathrm{~Hz}\right), 127.1,128.1$, $128.2,128.4\left(\mathrm{~d}, J_{\mathrm{C}-\mathrm{P}}=96.2 \mathrm{~Hz}\right), 129.9\left(\mathrm{~d}, J_{\mathrm{C}-\mathrm{P}}=9.2 \mathrm{~Hz}\right), 130.3\left(\mathrm{~d}, J_{\mathrm{C}-\mathrm{P}}=\right.$ $8.4 \mathrm{~Hz}), 134.2\left(\mathrm{~d}, J_{\mathrm{C}-\mathrm{P}}=1.8 \mathrm{~Hz}\right), 134.3\left(\mathrm{~d}, J_{\mathrm{C}-\mathrm{P}}=2.5 \mathrm{~Hz}\right), 137.8\left(\mathrm{~d}, J_{\mathrm{C}-\mathrm{P}}=\right.$ $12.7 \mathrm{~Hz}), 138.0\left(\mathrm{~d}, J_{\mathrm{C}-\mathrm{P}}=12.4 \mathrm{~Hz}\right), 143.7\left(\mathrm{~d}, J_{\mathrm{C}-\mathrm{P}}=5.9 \mathrm{~Hz}\right), 173.6$.

${ }^{31} \mathrm{P}$ NMR $\left(162 \mathrm{MHz}, \mathrm{CDCl}_{3}\right): \delta=31.3$.

HRMS: $m / z[\mathrm{M}+\mathrm{H}]$ calcd. for $\mathrm{C}_{26} \mathrm{H}_{28} \mathrm{NO}_{3} \mathrm{P}+\mathrm{H}$ : 434.1885; found: 434.1872.

\section{3-(Bis(3,5-dimethylphenyl)phosphinoyl)-3-hydroxy-1-benzylin-} dolin-2-one (3s)

The title compound was prepared by following the general procedure for Scheme 1, using 1-benzylisatin 1d $(0.5 \mathrm{mmol}, 0.118 \mathrm{~g})$ and bis (3,5-dimethylphenyl)phosphine oxide $2 \mathbf{c}(0.5 \mathrm{mmol}, 0.129 \mathrm{~g})$, providing 3s as a white solid. Yield: $0.227 \mathrm{~g} \mathrm{(92 \% );} \mathrm{mp} 134{ }^{\circ} \mathrm{C}$. 
${ }^{1} \mathrm{H}$ NMR $\left(400 \mathrm{MHz}, \mathrm{CDCl}_{3}\right): \delta=2.25(\mathrm{~s}, 6 \mathrm{H}), 2.26(\mathrm{~s}, 6 \mathrm{H}), 4.45$ and $4.79(\mathrm{ABq}, J=16.0 \mathrm{~Hz}, 2 \mathrm{H}), 6.52(\mathrm{~d}, J=7.6 \mathrm{~Hz}, 1 \mathrm{H}), 6.87(\mathrm{t}, J=7.6 \mathrm{~Hz}$, $1 \mathrm{H}), 6.92-6.97(\mathrm{~m}, 2 \mathrm{H}), 7.00(\mathrm{~d}, J=7.6 \mathrm{~Hz}, 1 \mathrm{H}), 7.13-7.22(\mathrm{~m}, 6 \mathrm{H})$, $7.28(\mathrm{~s}, 1 \mathrm{H}), 7.31(\mathrm{~s}, 1 \mathrm{H}), 7.42(\mathrm{~s}, 1 \mathrm{H}), 7.45(\mathrm{~s}, 1 \mathrm{H})$.

${ }^{13} \mathrm{C}$ NMR $\left(100 \mathrm{MHz}, \mathrm{CDCl}_{3}\right): \delta=21.36,21.38,44.3,79.8\left(\mathrm{~d}, J_{\mathrm{C}-\mathrm{P}}=\right.$ $70.3 \mathrm{~Hz}), 109.1,122.8,124.7,126.8\left(\mathrm{~d}, J_{\mathrm{C}-\mathrm{P}}=95.3 \mathrm{~Hz}\right), 126.9,127.0$, $127.4\left(\mathrm{~d}, J_{\mathrm{C}-\mathrm{P}}=93.0 \mathrm{~Hz}\right), 127.5,128.7,128.82,128.86,130.1\left(\mathrm{~d}, J_{\mathrm{C}-\mathrm{P}}=\right.$ $9.3 \mathrm{~Hz}$ ), 130.3 (d, $\left.J_{\mathrm{C}-\mathrm{P}}=9.0 \mathrm{~Hz}\right), 134.3\left(\mathrm{~d}, J_{\mathrm{C}-\mathrm{P}}=2.7 \mathrm{~Hz}\right), 134.5,135.0$, $137.8\left(\mathrm{~d}, J_{\mathrm{C}-\mathrm{P}}=12.6 \mathrm{~Hz}\right), 138.0\left(\mathrm{~d}, J_{\mathrm{C}-\mathrm{P}}=12.4 \mathrm{~Hz}\right), 143.8\left(\mathrm{~d}, J_{\mathrm{C}-\mathrm{P}}=\right.$ $5.8 \mathrm{~Hz}), 174.4$

${ }^{31} \mathrm{P}$ NMR $\left(162 \mathrm{MHz}, \mathrm{CDCl}_{3}\right): \delta=31.6$.

HRMS: $m / z[\mathrm{M}+\mathrm{H}]$ calcd. for $\mathrm{C}_{31} \mathrm{H}_{30} \mathrm{NO}_{3} \mathrm{P}+\mathrm{H}$ : 496.2042; found: 496.2051.

\section{3-(Bis(3,5-dimethylphenyl)phosphinoyl)-5-chloro-3-hydroxy-in- dolin-2-one (3t)}

The title compound was prepared by following the general procedure for Scheme 1, using 5-chloroisatin $1 e(0.5 \mathrm{mmol}, 0.090 \mathrm{~g})$ and bis (3,5dimethylphenyl)phosphine oxide $2 \mathrm{c}(0.5 \mathrm{mmol}, 0.129 \mathrm{~g})$, providing 3t as a white solid. Yield: $0.202 \mathrm{~g}(92 \%) ; \mathrm{mp} 208{ }^{\circ} \mathrm{C}$.

${ }^{1} \mathrm{H}$ NMR (400 MHz, DMSO- $\left.d_{6}\right): \delta=2.28(\mathrm{~s}, 6 \mathrm{H}), 2.33(\mathrm{~s}, 6 \mathrm{H}), 6.21(\mathrm{~s}$, $1 \mathrm{H}), 6.76(\mathrm{~d}, J=8.4 \mathrm{~Hz}, 1 \mathrm{H}), 7.18(\mathrm{~s}, 1 \mathrm{H}), 7.24-7.33(\mathrm{~m}, 3 \mathrm{H}), 7.46(\mathrm{~s}$, $1 \mathrm{H}), 7.49(\mathrm{~s}, 1 \mathrm{H}), 7.53(\mathrm{~s}, 1 \mathrm{H}), 7.56(\mathrm{~s}, 1 \mathrm{H}), 10.60(\mathrm{~s}, 1 \mathrm{H})$.

${ }^{13} \mathrm{C}$ NMR $\left(100 \mathrm{MHz}\right.$, DMSO- $\left.d_{6}\right): \delta=20.94,20.98,80.0$ (d, $J_{\mathrm{C}-\mathrm{P}}=$ $79.7 \mathrm{~Hz}), 110.7,124.8$ (d, $\left.J_{\mathrm{C}-\mathrm{P}}=2.4 \mathrm{~Hz}\right), 125.6$ (d, $\left.J_{\mathrm{C}-\mathrm{P}}=2.1 \mathrm{~Hz}\right), 128.9$, 129.4, $129.83\left(\mathrm{~d}, J_{\mathrm{C}-\mathrm{P}}=94.4 \mathrm{~Hz}\right), 129.88\left(\mathrm{~d}, J_{\mathrm{C}-\mathrm{P}}=8.2 \mathrm{~Hz}\right), 129.9(\mathrm{~d}$, $\left.J_{\text {C-P }}=5.9 \mathrm{~Hz}\right), 130.0\left(\mathrm{~d}, J_{\mathrm{C}-\mathrm{P}}=94.8 \mathrm{~Hz}\right), 133.2,133.6,136.8\left(\mathrm{~d}, J_{\mathrm{C}-\mathrm{P}}=\right.$ $12.3 \mathrm{~Hz}), 137.2\left(\mathrm{~d}, J_{\mathrm{C}-\mathrm{P}}=12.0 \mathrm{~Hz}\right), 141.6\left(\mathrm{~d}, J_{\mathrm{C}-\mathrm{P}}=5.7 \mathrm{~Hz}\right), 175.0$.

${ }^{31} \mathrm{P}$ NMR $\left(162 \mathrm{MHz}\right.$, DMSO- $\left.d_{6}\right): \delta=27.9$.

HRMS: $m / z[\mathrm{M}+\mathrm{H}]$ calcd. for $\mathrm{C}_{24} \mathrm{H}_{23} \mathrm{ClNO}_{3} \mathrm{P}+\mathrm{H}$ : 440.1182; found: 440.1172 .

\section{3-(Bis(3,5-dimethylphenyl)phosphinoyl)-5-bromo-3-hydroxy-in- dolin-2-one (3u)}

The title compound was prepared by following the general procedure for Scheme 1, using 5-bromoisatin $\mathbf{1 i}(0.5 \mathrm{mmol}, 0.113 \mathrm{~g})$ and bis (3,5dimethylphenyl)phosphine oxide $2 \mathrm{c}(0.5 \mathrm{mmol}, 0.129 \mathrm{~g})$, providing 3u as a white solid. Yield: $0.207 \mathrm{~g}(86 \%) ; \mathrm{mp} 178{ }^{\circ} \mathrm{C}$.

${ }^{1} \mathrm{H}$ NMR (400 MHz, DMSO- $d_{6}$ ): $\delta=2.24(\mathrm{~s}, 6 \mathrm{H}$ ), 2.28 (s, $6 \mathrm{H}$ ), 5.55 (d, $J=11.6 \mathrm{~Hz}, 1 \mathrm{H}), 6.74(\mathrm{~d}, J=8.0 \mathrm{~Hz}, 1 \mathrm{H}), 7.19(\mathrm{~s}, 1 \mathrm{H}), 7.23(\mathrm{~s}, 1 \mathrm{H})$, 7.27 (s, $1 \mathrm{H}), 7.30$ (s, $1 \mathrm{H}), 7.35(\mathrm{~s}, 1 \mathrm{H}), 7.39$ (d, J = 8.4 Hz, $1 \mathrm{H}), 7.51$ (s, $1 \mathrm{H}), 7.54(\mathrm{~s}, 1 \mathrm{H}), 10.7(\mathrm{~s}, 1 \mathrm{H})$

${ }^{13} \mathrm{C}$ NMR $\left(100 \mathrm{MHz}\right.$, DMSO- $\left.d_{6}\right): \delta=20.2,69.9\left(\mathrm{~d}, J_{\mathrm{C}-\mathrm{P}}=6.0 \mathrm{~Hz},\right), 111.5$, $112.8,127.1,128.1\left(\mathrm{~d}, J_{\mathrm{C}-\mathrm{P}}=8.3 \mathrm{~Hz}\right), 129.5\left(\mathrm{~d}, J_{\mathrm{C}-\mathrm{P}}=3.2 \mathrm{~Hz}\right), 130.7(\mathrm{~d}$, $\left.J_{\mathrm{C}-\mathrm{P}}=10.0 \mathrm{~Hz}\right), 132.3,132.6,133.5,137.6\left(\mathrm{~d}, J_{\mathrm{C}-\mathrm{P}}=14.0 \mathrm{~Hz}\right), 141.7$, 172.4 .

${ }^{31} \mathrm{P}$ NMR $\left(162 \mathrm{MHz}\right.$, DMSO- $\left.d_{6}\right): \delta=35.6$.

HRMS: $m / z[\mathrm{M}+\mathrm{H}]$ calcd. for $\mathrm{C}_{24} \mathrm{H}_{23} \mathrm{BrNO}_{3} \mathrm{P}+\mathrm{H}$ : 484.0677; found: 484.0689 .

\section{3-(Bis(3,5-dimethylphenyl)phosphinoyl)-5-chloro-3-hydroxy-1- ethylindolin-2-one (3v)}

The title compound was prepared by following the general procedure for Scheme 1, using 5-chloro-1-ethylisatin $1 \mathrm{~g}(0.5 \mathrm{mmol}, 0.105 \mathrm{~g})$ and bis (3,5-dimethylphenyl)phosphine oxide $2 \mathrm{c}(0.5 \mathrm{mmol}, 0.129 \mathrm{~g})$, providing $3 v$ as a white solid. Yield: $0.217 \mathrm{~g}(93 \%) ; \mathrm{mp} 138{ }^{\circ} \mathrm{C}$.
${ }^{1} \mathrm{H}$ NMR $\left(400 \mathrm{MHz}, \mathrm{CDCl}_{3}\right): \delta=0.87(\mathrm{t}, J=7.2 \mathrm{~Hz}, 3 \mathrm{H}), 2.29(\mathrm{~s}, 6 \mathrm{H})$, $2.33(\mathrm{~s}, 6 \mathrm{H}), 3.32-3.37(\mathrm{~m}, 1 \mathrm{H}), 3.56-3.61(\mathrm{~m}, 1 \mathrm{H}), 4.75(\mathrm{~d}, J=3.6 \mathrm{~Hz}$, $1 \mathrm{H}), 6.60(\mathrm{~d}, J=8.4 \mathrm{~Hz}, 1 \mathrm{H}), 6.85-6.90(\mathrm{~m}, 1 \mathrm{H}), 7.15(\mathrm{~s}, 1 \mathrm{H}), 7.21(\mathrm{~s}$, $1 \mathrm{H}), 7.25-7.27$ (m, $2 \mathrm{H}), 7.30$ (s, $1 \mathrm{H}), 7.33$ (s, $1 \mathrm{H}), 7.42(\mathrm{~s}, 1 \mathrm{H}), 7.45$ (s, $1 \mathrm{H})$.

${ }^{13} \mathrm{C}$ NMR $\left(100 \mathrm{MHz}, \mathrm{CDCl}_{3}\right): \delta=12.0,21.3,21.4,35.0,79.7$ (d, $J_{\mathrm{C}-\mathrm{P}}=$ $68.0 \mathrm{~Hz}), 108.8,126.2,126.4,127.5\left(\mathrm{~d}, J_{\mathrm{C}-\mathrm{P}}=2.7 \mathrm{~Hz}\right), 127.9\left(\mathrm{~d}, J_{\mathrm{C}-\mathrm{P}}=\right.$ $2.6 \mathrm{~Hz}), 128.2,128.6,130.0\left(\mathrm{~d}, J_{\mathrm{C}-\mathrm{P}}=9.4 \mathrm{~Hz}\right), 130.2\left(\mathrm{~d}, J_{\mathrm{C}-\mathrm{P}}=8.8 \mathrm{~Hz}\right)$, $134.5\left(\mathrm{~d}, J_{\mathrm{C}-\mathrm{P}}=2.4 \mathrm{~Hz}\right), 134.6\left(\mathrm{~d}, J_{\mathrm{C}-\mathrm{P}}=2.5 \mathrm{~Hz}\right), 137.9\left(\mathrm{~d}, J_{\mathrm{C}-\mathrm{P}}=12.6 \mathrm{~Hz}\right)$, $138.2\left(\mathrm{~d}, J_{\mathrm{C}-\mathrm{P}}=12.5 \mathrm{~Hz}\right), 142.1\left(\mathrm{~d}, J_{\mathrm{C}-\mathrm{P}}=5.6 \mathrm{~Hz}\right), 173.3$.

${ }^{31} \mathrm{P}$ NMR $\left(162 \mathrm{MHz}, \mathrm{CDCl}_{3}\right): \delta=31.4$.

HRMS: $m / z[\mathrm{M}+\mathrm{H}]$ calcd. for $\mathrm{C}_{26} \mathrm{H}_{27} \mathrm{ClNO}_{3} \mathrm{P}+\mathrm{H}$ : 468.1495; found: 468.1480 .

\section{3-(Bis(3,5-dimethylphenyl)phosphinoyl)-5-bromo-3-hydroxy-1- ethylindolin-2-one (3w)}

The title compound was prepared by following the general procedure for Scheme 1, using 5-bromo-1-ethylisatin $\mathbf{1 j}(0.5 \mathrm{mmol}, 0.127 \mathrm{~g})$ and bis (3,5-dimethylphenyl)phosphine oxide $2 \mathrm{c}(0.5 \mathrm{mmol}, 0.129 \mathrm{~g})$, providing 3w as a white solid. Yield: $0.240 \mathrm{~g}(94 \%) ; \mathrm{mp} 132{ }^{\circ} \mathrm{C}$.

${ }^{1} \mathrm{H}$ NMR (400 MHz, $\left.\mathrm{CDCl}_{3}\right): \delta=0.86(\mathrm{t}, J=7.2 \mathrm{~Hz}, 3 \mathrm{H}), 2.29(\mathrm{~s}, 6 \mathrm{H})$, $2.33(\mathrm{~s}, 6 \mathrm{H}), 3.30-3.36(\mathrm{~m}, 1 \mathrm{H}), 3.54-3.60(\mathrm{~m}, 1 \mathrm{H}), 5.14(\mathrm{~s}, 1 \mathrm{H}), 6.55$ $(\mathrm{d}, J=8.4 \mathrm{~Hz}, 1 \mathrm{H}), 7.01(\mathrm{~s}, 1 \mathrm{H}), 7.15(\mathrm{~s}, 1 \mathrm{H}), 7.26(\mathrm{~s}, 1 \mathrm{H}), 7.31(\mathrm{~s}, 1 \mathrm{H})$, 7.34 (s, $1 \mathrm{H}), 7.41-7.51(\mathrm{~m}, 3 \mathrm{H})$.

${ }^{13} \mathrm{C}$ NMR $\left(100 \mathrm{MHz}, \mathrm{CDCl}_{3}\right): \delta=12.0,21.3,21.4,35.0,79.8\left(\mathrm{~d}, J_{\mathrm{C}-\mathrm{P}}=\right.$ $68.3 \mathrm{~Hz}), 109.2,115.0,126.2,126.8,127.6\left(\mathrm{~d}, J_{\mathrm{C}-\mathrm{P}}=96.3 \mathrm{~Hz}\right), 130.0(\mathrm{~d}$, $\left.J_{C-P}=9.2 \mathrm{~Hz}\right), 130.3\left(\mathrm{~d}, J_{\mathrm{C}-\mathrm{P}}=9.1 \mathrm{~Hz}\right), 132.9,134.4\left(\mathrm{~d}, J_{\mathrm{C}-\mathrm{P}}=2.4 \mathrm{~Hz}\right)$, $134.5\left(\mathrm{~d}, J_{\mathrm{C}-\mathrm{P}}=2.5 \mathrm{~Hz}\right), 137.9\left(\mathrm{~d}, J_{\mathrm{C}-\mathrm{P}}=12.9 \mathrm{~Hz}\right), 138.1\left(\mathrm{~d}, J_{\mathrm{C}-\mathrm{P}}=\right.$ $12.5 \mathrm{~Hz}), 142.5\left(\mathrm{~d}, J_{\mathrm{C}-\mathrm{P}}=5.9 \mathrm{~Hz}\right), 173.2$.

${ }^{31} \mathrm{P}$ NMR $\left(162 \mathrm{MHz}, \mathrm{CDCl}_{3}\right): \delta=31.5$.

HRMS: $m / z[\mathrm{M}+\mathrm{H}]$ calcd. for $\mathrm{C}_{26} \mathrm{H}_{27} \mathrm{BrNO}_{3} \mathrm{P}+\mathrm{H}$ : 512.0990 ; found: 512.0982 .

\section{3-(Bis(3,5-dimethylphenyl)phosphinoyl)-3-hydroxy-5-nitro-indo- lin-2-one (3x)}

The title compound was prepared by following the general procedure for Scheme 1, using 5-nitroisatin $1 \mathbf{k}(0.5 \mathrm{mmol}, 0.096 \mathrm{~g})$ and bis (3,5dimethylphenyl)phosphine oxide $\mathbf{2 c}(0.5 \mathrm{mmol}, 0.129 \mathrm{~g})$, providing $3 \mathbf{x}$ as a white solid. Yield: $0.207 \mathrm{~g}(92 \%) ; \mathrm{mp} 184^{\circ} \mathrm{C}$.

${ }^{1} \mathrm{H}$ NMR (400 MHz, $\left.\mathrm{CDCl}_{3} / \mathrm{DMSO}_{6} \mathrm{~d}_{6}\right): \delta=2.36(\mathrm{~s}, 12 \mathrm{H}), 6.80-6.95(\mathrm{~m}$, $2 \mathrm{H}), 7.18$ (s, $1 \mathrm{H}), 7.27$ (s, $1 \mathrm{H}), 7.28-7.35(\mathrm{~m}, 1 \mathrm{H}), 7.50-7.60(\mathrm{~m}, 2 \mathrm{H})$, $7.68(\mathrm{~s}, 1 \mathrm{H}), 7.71(\mathrm{~s}, 1 \mathrm{H}), 8.08(\mathrm{t}, J=7.2 \mathrm{~Hz}, 1 \mathrm{H}), 10.7(\mathrm{~s}, 1 \mathrm{H})$.

${ }^{13} \mathrm{C}$ NMR $\left(100 \mathrm{MHz}\right.$, DMSO- $\left.d_{6}\right): \delta=20.9,21.0,79.7\left(\mathrm{~d}, J_{\mathrm{C}-\mathrm{P}}=78.4 \mathrm{~Hz}\right)$, $109.7,121.0,126.9,127.8,129.0,129.2\left(\mathrm{~d}, J_{\mathrm{C}-\mathrm{P}}=94.9 \mathrm{~Hz}\right), 129.9(\mathrm{~d}$, $\left.J_{\mathrm{C}-\mathrm{P}}=8.7 \mathrm{~Hz}\right), 133.5,133.9,137.1\left(\mathrm{~d}, J_{\mathrm{C}-\mathrm{P}}=12.3 \mathrm{~Hz}\right), 137.5\left(\mathrm{~d}, J_{\mathrm{C}-\mathrm{P}}=\right.$ $12.2 \mathrm{~Hz}), 141.3,149.1\left(\mathrm{~d}, J_{\mathrm{C}-\mathrm{p}}=5.3 \mathrm{~Hz}\right), 175.8$.

${ }^{31} \mathrm{P} \operatorname{NMR}\left(162 \mathrm{MHz}, \mathrm{CDCl}_{3}\right): \delta=28.4$.

HRMS: $m / z[\mathrm{M}+\mathrm{H}]$ calcd. for $\mathrm{C}_{24} \mathrm{H}_{23} \mathrm{~N}_{2} \mathrm{O}_{5} \mathrm{P}+\mathrm{H}$ : 451.1423; found: 451.1430 .

\section{3-Diphenylphosphoryl-3-hydroxy-indolin-2-one (3y1 $)^{20}$}

The title compound was prepared by following the general procedure for Scheme 1, using isatin 1a (1.0 mmol, $0.147 \mathrm{~g})$ and diphenyl phosphite $2 \mathbf{d}(1.0 \mathrm{mmol}, 0.234 \mathrm{~g}, 0.2 \mathrm{~mL})$, providing $\mathbf{3 y 1}$ as a white solid. Yield: $0.343 \mathrm{~g}(90 \%) ; \mathrm{mp} 130{ }^{\circ} \mathrm{C}$.

${ }^{1} \mathrm{H}$ NMR $\left(400 \mathrm{MHz}, \mathrm{CDCl}_{3} /\right.$ DMSO- $\left._{6}\right): \delta=6.25(\mathrm{bs}, 1 \mathrm{H}), 6.84-6.93(\mathrm{~m}$, $3 \mathrm{H}), 7.02-7.10(\mathrm{~m}, 2 \mathrm{H}), 7.13-7.33(\mathrm{~m}, 8 \mathrm{H}), 7.65(\mathrm{~d}, J=7.6 \mathrm{~Hz}, 1 \mathrm{H})$, $9.85(\mathrm{~s}, 1 \mathrm{H})$. 
${ }^{13} \mathrm{C}$ NMR $\left(100 \mathrm{MHz}, \mathrm{CDCl}_{3} / \mathrm{DMSO}-d_{6}\right): \delta=75.5,110.3,120.4\left(\mathrm{~d}, J_{\mathrm{C}-\mathrm{P}}=\right.$ $3.8 \mathrm{~Hz}), 120.9\left(\mathrm{~d}, J_{\mathrm{C}-\mathrm{P}}=3.9 \mathrm{~Hz}\right), 122.4,125.0\left(\mathrm{~d}, J_{\mathrm{C}-\mathrm{P}}=9.2 \mathrm{~Hz}\right), 125.2$, $126.8\left(\mathrm{~d}, J_{\mathrm{C}-\mathrm{P}}=3.4 \mathrm{~Hz}\right), 129.3\left(\mathrm{~d}, J_{\mathrm{C}-\mathrm{P}}=8.3 \mathrm{~Hz}\right), 130.5,142.4\left(\mathrm{~d}, J_{\mathrm{C}-\mathrm{P}}=\right.$ $9.1 \mathrm{~Hz}), 150.1\left(\mathrm{~d}, J_{\mathrm{C}-\mathrm{P}}=10.2 \mathrm{~Hz}\right), 150.4\left(\mathrm{~d}, J_{\mathrm{C}-\mathrm{P}}=10.4 \mathrm{~Hz}\right), 174.6(\mathrm{~d}$, $\left.J_{\mathrm{C}-\mathrm{P}}=4.9 \mathrm{~Hz}\right)$.

${ }^{31} \mathrm{P}$ NMR $\left(162 \mathrm{MHz}, \mathrm{CDCl}_{3} / \mathrm{DMSO}_{-} d_{6}\right): \delta=9.1$.

HRMS: $m / z[\mathrm{M}+\mathrm{Na}]$ calcd. for $\mathrm{C}_{20} \mathrm{H}_{16} \mathrm{NO}_{5} \mathrm{P}+\mathrm{Na}$ : 404.0664; found: 404.0622.

\section{3-Diphenylphosphoryl-3-hydroxy-1-methylindolin-2-one (3y2)}

The title compound was prepared by following the general procedure for Scheme 1, using $N$-methylisatin $\mathbf{1 b}(1.0 \mathrm{mmol}, 0.161 \mathrm{~g})$ and diphenyl phosphite $\mathbf{2 d}(1.0 \mathrm{mmol}, 0.234 \mathrm{~g}, 0.2 \mathrm{~mL})$, providing $3 \mathbf{y} 2$ as a white solid. Yield: $0.367 \mathrm{~g}(93 \%) ; \mathrm{mp} 106{ }^{\circ} \mathrm{C}$.

${ }^{1} \mathrm{H} \mathrm{NMR}\left(400 \mathrm{MHz}, \mathrm{CDCl}_{3} / \mathrm{DMSO}_{6}\right): \delta=3.22(\mathrm{~s}, 3 \mathrm{H}), 6.82-6.90(\mathrm{~m}$, $3 \mathrm{H}), 7.07-7.12$ (m, $2 \mathrm{H}), 7.15-7.25$ (m, $5 \mathrm{H}), 7.27-7.35$ (m, $2 \mathrm{H}), 7.36-$ $7.42(\mathrm{~m}, 1 \mathrm{H}), 7.67(\mathrm{~d}, J=7.6 \mathrm{~Hz}, 1 \mathrm{H})$.

${ }^{13} \mathrm{C}$ NMR (100 MHz, $\mathrm{CDCl}_{3} /$ DMSO- $_{6}$ ): $\delta=26.8,75.2,108.8,120.4$ (d, $\left.J_{C-P}=3.9 \mathrm{~Hz}\right), 120.7\left(\mathrm{~d}, J_{\mathrm{C}-\mathrm{P}}=4.1 \mathrm{~Hz}\right), 123.5\left(\mathrm{~d}, J_{\mathrm{C}-\mathrm{P}}=2.5 \mathrm{~Hz}\right), 125.5(\mathrm{~d}$, $\left.J_{C-P}=11.4 \mathrm{~Hz}\right), 126.9\left(\mathrm{~d}, J_{\mathrm{C}-\mathrm{P}}=3.4 \mathrm{~Hz}\right), 129.7\left(\mathrm{~d}, J_{\mathrm{C}-\mathrm{P}}=13.4 \mathrm{~Hz}\right), 131.1$, $144.4\left(\mathrm{~d}, J_{\mathrm{C}-\mathrm{P}}=8.8 \mathrm{~Hz}\right), 150.1\left(\mathrm{~d}, J_{\mathrm{C}-\mathrm{P}}=9.8 \mathrm{~Hz}\right), 150.3\left(\mathrm{~d}, J_{\mathrm{C}-\mathrm{P}}=10.3 \mathrm{~Hz}\right)$, $173.0\left(\mathrm{~d}, J_{\mathrm{C}-\mathrm{P}}=6.0 \mathrm{~Hz}\right)$.

${ }^{31} \mathrm{P} \mathrm{NMR}\left(162 \mathrm{MHz}, \mathrm{CDCl}_{3} / \mathrm{DMSO}_{-} d_{6}\right): \delta=8.8$.

HRMS: $m / z[\mathrm{M}+\mathrm{H}]$ calcd. for $\mathrm{C}_{21} \mathrm{H}_{18} \mathrm{NO}_{5} \mathrm{P}+\mathrm{H}$ : 396.1001; found: 396.0989 .

\section{3-Diphenylphosphoryl-3-hydroxy-1-ethylindolin-2-one (3y3)}

The title compound was prepared by following the general procedure for Scheme 1, using $N$-ethylisatin $1 c$ ( $1.0 \mathrm{mmol}, 0.175 \mathrm{~g})$ and diphenyl phosphite $2 \mathbf{d}$ ( $1.0 \mathrm{mmol}, 0.234 \mathrm{~g}, 0.2 \mathrm{~mL}$ ), providing $\mathbf{3 y 3}$ as a colorless viscous liquid. Yield: $0.372 \mathrm{~g}(91 \%)$.

${ }^{1} \mathrm{H}$ NMR (400 MHz, $\left.\mathrm{CDCl}_{3}\right): \delta=1.27(\mathrm{t}, J=7.2 \mathrm{~Hz}, 3 \mathrm{H}), 3.73-3.76(\mathrm{~m}$, $2 \mathrm{H}), 5.78(\mathrm{~d}, J=12.0 \mathrm{~Hz}, 1 \mathrm{H}), 6.83(\mathrm{~d}, J=8.0 \mathrm{~Hz}, 1 \mathrm{H}), 6.98(\mathrm{t}, J=$ 7.6 Hz, $1 \mathrm{H}), 7.18-7.24(\mathrm{~m}, 5 \mathrm{H}), 7.25-7.47$ (m, $7 \mathrm{H})$.

${ }^{13} \mathrm{C} \mathrm{NMR}\left(100 \mathrm{MHz}, \mathrm{CDCl}_{3}\right): \delta=12.5,35.0,73.6\left(\mathrm{~d}, J_{\mathrm{C}-\mathrm{P}}=6.0 \mathrm{~Hz}\right), 108.7$, $120.50\left(\mathrm{~d}, J_{\mathrm{C}-\mathrm{P}}=4.7 \mathrm{~Hz}\right), 120.59\left(\mathrm{~d}, J_{\mathrm{C}-\mathrm{P}}=4.7 \mathrm{~Hz}\right), 123.0,123.6\left(\mathrm{~d}, J_{\mathrm{C}-\mathrm{P}}=\right.$ $3.2 \mathrm{~Hz}), 125.6,126.3,129.8,130.9,143.4,150.3\left(\mathrm{~d}, J_{\mathrm{C}-\mathrm{P}}=7.3 \mathrm{~Hz}\right), 150.5$ $\left(\mathrm{d}, J_{\mathrm{C}-\mathrm{P}}=7.4 \mathrm{~Hz}\right), 170.7$.

${ }^{31} \mathrm{P}$ NMR $\left(162 \mathrm{MHz}, \mathrm{CDCl}_{3}\right): \delta=10.7$.

HRMS: $m / z[\mathrm{M}+\mathrm{H}]$ calcd. for $\mathrm{C}_{22} \mathrm{H}_{20} \mathrm{NO}_{5} \mathrm{P}+\mathrm{H}$ : 410.1157; found: 410.1168 .

\section{3-Diphenylphosphoryl-5-chloro-3-hydroxy-1-methylindolin-2- one (3y4)}

The title compound was prepared by following the general procedure for Scheme 1, using 5-chloro-1-ethylisatin $\mathbf{1 g}(1.0 \mathrm{mmol}, 0.209 \mathrm{~g})$ and diphenyl phosphite $2 \mathbf{d}(1.0 \mathrm{mmol}, 0.234 \mathrm{~g}, 0.2 \mathrm{~mL})$, providing $3 \mathbf{y} 4$ as a colorless viscous liquid. Yield: $0.381 \mathrm{~g}(86 \%)$.

${ }^{1} \mathrm{H}$ NMR $\left(400 \mathrm{MHz}, \mathrm{CDCl}_{3}\right): \delta=1.25(\mathrm{t}, J=7.2 \mathrm{~Hz}, 3 \mathrm{H}), 3.69-3.75(\mathrm{~m}$, $2 \mathrm{H}), 5.73(\mathrm{~d}, J=12.4 \mathrm{~Hz}, 1 \mathrm{H}), 6.74(\mathrm{~d}, J=8.4 \mathrm{~Hz}, 1 \mathrm{H}), 7.06-7.08(\mathrm{~m}$, $1 \mathrm{H}), 7.23-7.26(\mathrm{~m}, 5 \mathrm{H}), 7.27-7.47(\mathrm{~m}, 6 \mathrm{H})$.

${ }^{13} \mathrm{C} \mathrm{NMR}\left(100 \mathrm{MHz}, \mathrm{CDCl}_{3}\right): \delta=12.4,35.2,73.1\left(\mathrm{~d}, J_{\mathrm{C}-\mathrm{P}}=5.9 \mathrm{~Hz}\right), 109.6$, $120.6\left(\mathrm{~d}, J_{\mathrm{C}-\mathrm{P}}=3.0 \mathrm{~Hz}\right), 125.1\left(\mathrm{~d}, J_{\mathrm{C}-\mathrm{P}}=2.9 \mathrm{~Hz}\right), 125.9\left(\mathrm{~d}, J_{\mathrm{C}-\mathrm{P}}=18.6 \mathrm{~Hz}\right)$, 126.7, 128.4, $129.9\left(\mathrm{~d}, J_{\mathrm{C}-\mathrm{P}}=3.2 \mathrm{~Hz}\right), 130.7,141.9,150.1$ (d, $J_{\mathrm{C}-\mathrm{P}}=$ $7.2 \mathrm{~Hz}), 150.4\left(\mathrm{~d}, J_{\mathrm{C}-\mathrm{P}}=7.6 \mathrm{~Hz}\right), 170.4\left(\mathrm{~d}, J_{\mathrm{C}-\mathrm{P}}=6.8 \mathrm{~Hz}\right)$.

${ }^{31} \mathrm{P}$ NMR $\left(162 \mathrm{MHz}, \mathrm{CDCl}_{3}\right): \delta=10.6$.
HRMS: $m / z[\mathrm{M}+\mathrm{Na}]$ calcd. for $\mathrm{C}_{22} \mathrm{H}_{19} \mathrm{ClNO}_{5} \mathrm{P}+\mathrm{Na}$ : 466.0587; found: 466.0542 .

\section{Funding Information}

The authors thank SERB-DST, New Delhi (YSS/2015/001870), DSTNew Delhi for INSPIRE Faculty Award (IFA-2014/CH-167) and the Council of Scientific\& Industrial Research-India (02(0341)/18/EMRII).

\section{Acknowledgment}

R.C. and P.S. acknowledge the UGC-India for Fellowships. R.B. thanks the SERB-DST for financial assistantship. The authors are grateful for MRC-MNIT Jaipur and USIC University of Rajasthan, Jaipur for spectroscopic analyses.

\section{Supporting Information}

Supporting information for this article is available online at https://doi.org/10.1055/s-0037-1610167.

\section{References and Notes}

(1) (a) Quin, L. D. A Guide to Organophosphorus Chemistry; Wiley Interscience: New York, 2000. (b) Tang, W.; Zhang, X. Chem. Rev. 2003, 103, 3029. (c) Montchamp, J.-L. Acc. Chem. Res. 2014, 47, 77. (d) Redmore, D. Chem. Rev. 1971, 71, 315. (e) Van der Jeught, S.; Stevens, C. V. Chem. Rev. 2009, 109, 2672. (f) Romero-Nieto, C.; Lýpez-Andarias, A.; Egler-Lucas, C.; Gebert, F.; Neus, J.-P.; Pilgram, O. Angew. Chem. Int. Ed. 2015, 54, 15872.

(2) (a) Phosphorus Ligands in Asymmetric Catalysis; Borner, A., Ed.; Wiley: New York, 2008. (b) P-Stereogenic Ligands in Enantioselective Catalysis; Grabulosa, A., Ed.; Royal Society of Chemistry: Cambridge, 2011.

(3) (a) Demkowicz, S.; Rachon, J.; Dasko, M.; Kozak, W. RSC Adv. 2016, 6, 7101. (b) Mucha, A.; Kafarski, P.; Berlicki, L. J. Med. Chem. 2011, 54, 5955.

(4) van Berkel, S. S.; van Eldijk, M. B.; van Hest, J. C. M. Angew. Chem. Int. Ed. 2011, 50, 8806

(5) (a) Wang, C.; Fukazawa, A.; Taki, M.; Sato, Y.; Higashiyama, T.; Yamaguchi, S. Angew. Chem. Int. Ed. 2015, 54, 15213. (b) Queffelec, C.; Petit, M.; Janvier, P.; Knight, D. A.; Bujoli, B. Chem. Rev. 2012, 112, 3777. (c) Baumgartner, T.; Reau, R. Chem. Rev. 2006, 106, 4681.

(6) (a) Allen, M. C.; Fuhrer, W.; Tuck, B.; Wade, R.; Wood, J. M. J. Med. Chem. 1989, 32, 1652. (b) Miller, D. J.; Hammond, S. M.; Anderluzzi, D.; Bugg, T. D. H. J. Chem. Soc., Perkin Trans. 1 1998, 131. (c) Peyman, A.; Stahl, W.; Wagner, K.; Ruppert, D.; Budt, K. H. Bioorg. Med. Chem. Lett. 1994, 4, 2601. (d) Atherton, F. R.; Hassal, C. H.; Lambert, R. W. J. Med. Chem. 1986, 29, 29. (e) Emsley, J.; Hall, D. The Chemistry of Phosphorus; Harper \& Row: London, 1976, 494. (f) Meyer, J. H.; Barlett, P. A. J. Am. Chem. Soc. 1998, 120, 4600. (g) Maier, L.; Spoerri, H. Phosphorus, Sulfur Silicon Relat. Elem. 1991, 61, 69.

(7) (a) Jablonkai, E.; Keglevich, G. Org. Prep. Proced. Int. 2014, 46, 281. (b) Hirao, T.; Masunaga, T.; Ohshiro, Y.; Agawa, T. Tetrahedron Lett. 1980, 21, 3595. (c) Hirao, T.; Masunaga, T.; Yamada, N.; Ohshiro, Y.; Agawa, T. Bull. Chem. Soc. Jpn. 1982, 55, 909. 
(d) Hirao, T.; Masunaga, T.; Ohshiro, Y.; Agawa, T. Synthesis 1981, 56. (e) Schwan, A. L. Chem. Soc. Rev. 2004, 33, 218. (f) Prim, D.; Campagne, J. M.; Joseph, D.; Andrioletti, B. Tetrahedron 2002, 58, 2041.

(8) (a) Kohno, J.; Koguchi, Y.; Nishio, M.; Nakao, K.; Juroda, M.; Shimizu, R.; Ohnuki, T.; Komatsubara, S. J. Org. Chem. 2000, 65, 990. (b) Toshinori, K.; Shizuka, S.; Hideyuki, S.; Ayumi, O.; Haruaki, I.; Takaaki, K.; Juńichi, K. J. Nat. Prod. 2006, 69, 1517. (c) Tokunaga, T.; Hume, W. E.; Umezome, T.; Okazaki, K.; Ueki, Y.; Kumagai, K.; Hourai, S.; Nagamine, J.; Seki, H.; Taiji, M.; Noguchi, H.; Nagata, R. J. Med. Chem. 2001, 44, 4641. (d) Galliford, C. V.; Scheidt, K. A. Angew. Chem. Int. Ed. 2007, 46, 8748. (e) Stratmann, K.; Moore, R. E.; Bonjouklian, R.; Deeter, J. B.; Patterson, G. M. L.; Shaffer, S.; Smith, C. D.; Smitka, T. A. J. Am. Chem. Soc. 1994, 116, 9935. (f) Rasmussen, H. B.; Macleod, J. K. J. Nat. Prod. 1997, 60, 1152. (g) Tokunaga, T.; Hume, W. E.; Nagamine, J.; Kawamura, T.; Taiji, M.; Nagata, R. Bioorg. Med. Chem. Lett. 2005, 15, 1789. (h) Edmondson, S. D.; Danishefsky, S. J. Angew. Chem. Int. Ed. 1998, 37, 1138. (i) Lerchner, A.; Carreira, E. M. J. Am. Chem. Soc. 2002, 124, 14826.

(9) (a) Braude, F.; Lindwall, H. G. J. Am. Chem. Soc. 1933, 55, 325. (b) Luppi, G.; Cozzi, P. G.; Monari, M.; Kaptein, B.; Broxterman, Q. B.; Tomasini, C. J. Org. Chem. 2005, 70, 7418. (c) Guo, Q.; Bhanushali, M.; Zhao, C. G. Angew. Chem. Int. Ed. 2010, 49, 9460. (d) Marshall, J. A. Chem. Rev. 2000, 100, 3163. (e) Denmark, S. E.; Fu, J. Chem. Rev. 2003, 103, 2763. (f) Shintani, R.; Inoue, M.; Hayashi, T. Angew. Chem. Int. Ed. 2006, 45, 3353.

(10) (a) Yu, R.; Chen, X.; Martin, S. F.; Wang, Z. Org. Lett. 2017, 19, 1808. (b) Fu, T.; Qiao, H.; Peng, Z.; Hu, G.; Wu, X.; Gao, Y.; Zhao, Y. Org. Biomol. Chem. 2014, 12, 2895. (c) Berrino, R.; Cacchi, S.; Fabrizi, G.; Goggiamani, A.; Stabile, P. Org. Biomol. Chem. 2010, 8, 4518. (d) Zhang, J. S.; Chen, T.; Yang, J.; Han, L. B. Chem. Commun. 2015, 7540. (e) He, Y.; Wu, H.; Toste, F. D. Chem. Sci. 2015, 6, 1194. (f) Zhuang, R.; Xu, J.; Cai, Z.; Tang, G.; Fang, M.; Zhao, Y. Org. Lett. 2011, 13, 2110. (g) Petrakis, K. S.; Nagabhushan, T. L. J. Am. Chem. Soc. 1987, 109, 2831. (h) Zhao, Y. L.; Wu, G. J.; Han, F. S. Chem. Commun. 2012, 5868. (i) Yang, J.; Xiao, J.; Chen, T.; Yin, S. F.; Han, L. B. Chem. Commun. 2016, 12233. (j) Wang, T.; Sang, S.; Liu, L.; Qiao, H.; Gao, Y.; Zhao, Y. J. Org. Chem. 2014, 79, 608. (k) Yang, J.; Chen, T.; Han, L.-B. J. Am. Chem. Soc. 2015, 137, 1782. (l) Hu, G.; Chen, W.; Fu, T.; Peng, Z.; Qiao, H.; Gao, Y.; Zhao, Y. Org. Lett. 2013, 15, 5362. (m) Andaloussi, M.; Lindh, J.; Savmarker, J.; Sjoberg, P. J. R.; Larhed, M. Chem. Eur. J. 2009, 15, 13069. (n) Xu, W.; Hu, G.; Xu, P.; Gao, Y.; Yin, Y.; Zhao, Y. Adv. Synth. Catal. 2014, 356, 2948. (o) Yang, J.; Xiao, J.; Chen, T.; Han, L. B. J. Org. Chem. 2016, 81, 3911. (p) Miao, T.; Wang, L. Adv. Synth. Catal. 2014, 356, 967. (q) Luo, H.; Liu, H.; Chen, X.; Wang, K.; Luo, X.; Wang, K. Chem. Commun. 2017, 956. (r) Liu, C.; Szostak, M. Angew. Chem. Int. Ed. 2017, 56, 12718. (s) Fu, W. C.; So, C. M.; Kwong, F. Y. Org. Lett. 2015, 17, 5906.

(11) (a) Li, C.; Yano, T.; Ishida, N.; Murakami, M. Angew. Chem. Int. Ed. 2013, 52, 9801. (b) Hong, G.; Mao, D.; Wu, S.; Wang, L. J. Org. Chem. 2014, 79, 10629. (c) Feng, C. G.; Ye, M.; Xiao, K. J.; Li, S.; Yu, J. Q. J. Am. Chem. Soc. 2013, 135, 9322.

(12) (a) Zhou, A. X.; Mao, L. L.; Wang, G. W.; Yang, S. D. Chem. Commun. 2014, 8529. (b) Zhu, Y.; Chen, T.; Li, S.; Shimada, S.; Han, L. B. J. Am. Chem. Soc. 2016, 138, 5825. (c) Gao, M.; Li, Y.; Xie, F.; Chauvin, R.; Cui, X. Chem. Commun. 2016, 2846. (d) Wang, T.; Chen, S.; Shao, A.; Gao, M.; Huang, Y.; Lei, A. Org. Lett. 2015, 17, 118. (e) Zhang, H. J.; Lin, W.; Wu, Z.; Ruan, W.;
Wen, T. B. Chem. Commun. 2015, 3450. (f) Hou, C.; Ren, Y.; Lang, R.; Hu, X.; Xia, C.; Li, F. Chem. Commun. 2012, 5181. (g) Kuninobu, Y.; Yoshida, T.; Takai, K. J. Org. Chem. 2011, 76, 7370. (h) Baslé, O.; Li, C.-J. Chem. Commun. 2009, 4124. (i) Ke, J.; Tang, Y.; Yi, H.; Li, Y.; Cheng, Y.; Liu, C.; Lei, A. Angew. Chem. Int. Ed. 2015, 54, 6604. (j) Yang, J.; Chen, T.; Zhou, Y.; Yin, S.; Han, L. B. Chem. Commun. 2015, 3549. (k) Huo, C.; Wang, C.; Wu, M.; Jia, X.; Wang, X.; Yuan, Y.; Xie, H. Org. Biomol. Chem. 2014, 12, 3123. (l) Kagayama, T.; Nakano, A.; Sakaguchi, S.; Ishii, Y. Org. Lett. 2006, 8, 407.

(13) (a) Lenker, H. K.; Richard, M. E.; Reese, K. P.; Carter, A. F.; Zawisky, J. D.; Winter, E. F.; Bergeron, T. W.; Guydon, K. S.; Stockland, R. A. Jr J. Org. Chem. 2012, 77, 1378. (b) Hans, M.; Delaude, L.; Rodriguez, J.; Coquerel, Y. J. Org. Chem. 2014, 79, 2758. (c) Carlone, A.; Bartoli, G.; Bosco, M.; Sambri, L.; Melchiorre, P. Angew. Chem. Int. Ed. 2007, 46, 4504. (d) Yang, X.Y.; Tay, W. S.; Li, Y.; Pullarkat, S. A.; Leung, P.-H. Organometallics 2015, 34, 5196. (e) Guo, H.; Yoshimura, A.; Chen, T.; Saga, Y.; Han, L.-B. Green Chem. 2017, 19, 1502. (f) Hirai, T.; Han, L.-B. Org. Lett. 2007, 9, 53. (g) Peng, P.; Lu, Q.; Peng, L.; Liu, C.; Wang, G.; Lei, A. Chem. Commun. 2016, 12338.

(14) (a) Deng, T.; Wang, H.; Cai, C. Org. Biomol. Chem. 2014, 12, 5843. (b) Srinivas, V.; Balaraman, E.; Sajna, K. V.; Swamy, K. C. K. Eur. J. Org. Chem. 2011, 4222.

(15) (a) Peng, L.; Wang, I. L.; Bai, J. F.; Jia, L. N.; Yang, Q. C.; Huang, Q. C.; Xu, X. Y.; Wang, L. X. Tetrahedron Lett. 2011, 52, 1157. (b) Gurevich, P. A.; Akhmetova, G. Z.; Gubaidullin, A. T.; Moskva, V. V.; Litvinov, I. N. A. Russ. J. Gen. Chem. 1998, 68, 1501. (c) Shankar, J.; Karnakar, K.; Srinivas, B.; Nageswar, Y. V. D. Tetrahedron Lett. 2010, 51, 3938. (d) Molleti, N.; Kang, J. Y. Org. Biomol. Chem. 2016, 14, 8952. (e) Nazish, M.; Jakhar, A.; Khan, N. H.; Verma, S.; Kureshy, R. I.; Abdi, S. H. R.; Bajaj, H. C. Appl. Catal., A 2016, 515, 116.

(16) (a) Hosseini-Sarvari, M.; Tavakolian, M. Can. J. Chem. 2013, 91, 1117. (b) Jang, H. S.; Kim, Y.; Kim, D. Y. Beilstein J. Org. Chem. 2016, 12, 1551. (c) Shankar, J.; Karnakar, K.; Srinivas, B.; Nageswar, Y. V. D. Tetrahedron Lett. 2010, 51, 3938. (d) Nagarapu, L.; Mallepalli, R.; Kumar, U. N.; Venkateswarlu, P.; Bantu, R.; Yeramanchi, L. Tetrahedron Lett. 2012, 53, 1699. (e) Nazish, M.; Saravanan, S.; Khan, N. H.; Kumari, P.; Kureshy, R. I.; Abdi, S. H. R.; Bajaj, H. C. ChemPlusChem 2014, 79, 1753. (f) Liu, C.; Zhang, Y.; Qian, Q.; Yuan, D.; Yao, Y. Org. Lett. 2014, $16,6172$.

(17) (a) Choudhary, R.; Bai, R.; Singh, P.; Sharma, M. C.; Badsara, S. S. Tetrahedron 2017, 73, 4323. (b) Singh, P.; Bai, R.; Choudhary, R.; Sharma, M. C.; Badsara, S. S. RSC Adv. 2017, 7, 30594. (c) Bai, R.; Choudhary, R.; Singh, P.; Thakuria, R.; Sharma, M. C.; Badsara, S. S. ChemistrySelect 2018, 3, 3221.

(18) Garden, S. J.; Torres, J. C.; de Silva, L. E.; Pinto, A. C. Synth. Commun. 1998, 28, 1679.

(19) (a) Reactivity of P-H Group of Phosphorus Based Compounds, Chapter 3, 14 Troev, K. D., Ed.; Elsevier: Amsterdam, 2018. (b) Xie, J.; Li, H.; Xue, Q.; Cheng, Y.; Zhu, C. Adv. Synth. Catal. 2012, 354, 1646. (c) Han, W.; Mayer, P.; Ofial, A. R. Adv. Synth. Catal. 2010, 352, 1667. (d) Copey, L.; Jean-Gerard, L.; Andrioletti, B.; Framery, E. Tetrahedron Lett. 2016, 57, 543. (e) Wen, L.-R.; Sun, Y.-X.; Zhang, J.-W.; Guo, W. S.; Li, M. Green Chem. 2018, 20, 125.

(20) Hosseini-Sarvari, M.; Tavakolian, M. Can. J. Chem. 2013, 91, 1117. 